Supporting Information

Mechanistic investigations on the competition between the cyclopolymerization and $[2+2+2]$ cycloaddition of 1,6 -heptadiyne derivatives using second-generation Grubbs catalysts

Eun-Hye Kang, Cheol Kang, Sanghee Yang, Elina Oks, Tae-Lim Choi*

Department of Chemistry, Seoul National University, Seoul 08826, Korea

E-mail: tlc@snu.ac.kr 


\section{Characterization of polymers}

The spectroscopic data of $\mathbf{2 a},{ }^{1} \mathbf{2} \mathbf{a}^{,},{ }^{2} \mathbf{2} \mathbf{b},{ }^{3} \mathbf{2} \mathbf{c},{ }^{2} \mathbf{2 d},{ }^{2} \mathbf{2} \mathbf{g}^{2}$, poly $(\mathbf{5 a})^{4}$ and poly $(\mathbf{5 b})^{5}$ were reported in the literature.

2e: ${ }^{1} \mathrm{H}$ NMR (500 MHz, $\left.\mathrm{CD}_{2} \mathrm{Cl}_{2}\right) \delta 6.90-6.07$ (br, 2H), 3.42 (br, 5H), $3.15-2.52$ (br, 4H), $1.39-0.94$ (br, $\left.6 \mathrm{H}\right) ;{ }^{13} \mathrm{C}$ NMR (125 MHz, $\left.\mathrm{CD}_{2} \mathrm{Cl}_{2}\right) \delta 174.61,138.96,123.57,42.62,40.91,38.66,38.22,15.23,13.46$.

2f: ${ }^{1} \mathrm{H}$ NMR (500 MHz, $\left.\mathrm{CDCl}_{3}\right) \delta 7.13-5.83(\mathrm{br}, 2 \mathrm{H}), 4.34-3.69$ (br, 2H), 3.14 (br, 4H), 1.71 (br, 2H), 1.27 (br, $14 \mathrm{H}), 0.87(\mathrm{~d}, J=6.6 \mathrm{~Hz}, 3 \mathrm{H}) ;{ }^{13} \mathrm{C} \mathrm{NMR}\left(125 \mathrm{MHz}, \mathrm{CDCl}_{3}\right) \delta 175.86,138.30,123.28,65.19,40.43,37.37,32.02$, $29.68,29.43,29.40,28.81,26.03,22.80,14.23$.

2h: ${ }^{1} \mathrm{H}$ NMR (500 MHz, $\left.\mathrm{CDCl}_{3}\right) \delta 7.00-5.96$ (br, 2H), $3.95-3.29$ (br, 2H), $3.04-1.80$ (br, 5H), $1.47-0.50$ (br, $21 \mathrm{H}) ;{ }^{13} \mathrm{C}$ NMR $\left(125 \mathrm{MHz}, \mathrm{CDCl}_{3}\right) \delta 139.17,123.27,67.60,38.30,36.55,18.22,12.25$.

\section{Characterization of dimers (3) and trimers (4)}

The spectroscopic data of $\mathbf{3 a}^{6}$ and $\mathbf{3} \mathbf{c}^{7}$ were reported in the literature.

4a: ${ }^{1} \mathrm{H}$ NMR (500 MHz, CDCl $) \delta 7.08(\mathrm{~d}, J=7.6 \mathrm{~Hz}, 2 \mathrm{H}), 6.96(\mathrm{~d}, J=10.3 \mathrm{~Hz}, 4 \mathrm{H}), 4.19(\mathrm{q}, J=7.1 \mathrm{~Hz}, 8 \mathrm{H}), 4.08$ (q, $J=7.1 \mathrm{~Hz}, 4 \mathrm{H}), 3.54(\mathrm{~d}, J=3.0 \mathrm{~Hz}, 8 \mathrm{H}), 3.15(\mathrm{~s}, 4 \mathrm{H}), 1.25(\mathrm{t}, J=7.1 \mathrm{~Hz}, 12 \mathrm{H}), 1.14(\mathrm{t}, J=7.1 \mathrm{~Hz}, 6 \mathrm{H}) ;{ }^{13} \mathrm{C}$ $\operatorname{NMR}\left(125 \mathrm{MHz}, \mathrm{CDCl}_{3}\right) \delta 171.76,171.13,140.16,138.76,135.22,129.01,126.08,124.01,61.82,61.32,60.61$, 60.42, 40.55, 40.33, 39.17, 14.17, 14.03; HRMS (ESI): $\mathrm{m} / \mathrm{z}$ for $\mathrm{C}_{39} \mathrm{H}_{48} \mathrm{NaO}_{12}[\mathrm{M}+\mathrm{Na}]^{+}$, calcd. 731.3038 , found: 731.3040.

3b: ${ }^{1} \mathrm{H}$ NMR $\left(500 \mathrm{MHz}, \mathrm{CDCl}_{3}\right) \delta 7.07(\mathrm{~d}, J=7.6 \mathrm{~Hz}, 1 \mathrm{H}), 6.99(\mathrm{~s}, 1 \mathrm{H}), 6.94(\mathrm{~d}, J=7.7 \mathrm{~Hz}, 1 \mathrm{H}), 4.19-4.05(\mathrm{~m}$, $8 \mathrm{H}), 3.53(\mathrm{~s}, 4 \mathrm{H}), 3.34(\mathrm{~s}, 2 \mathrm{H}), 2.66(\mathrm{~d}, J=2.0 \mathrm{~Hz}, 2 \mathrm{H}), 2.12(\mathrm{t}, J=2.2 \mathrm{~Hz}, 1 \mathrm{H}), 1.66-1.57(\mathrm{~m}, 8 \mathrm{H}), 1.29(\mathrm{~d}, J=$ $4.5 \mathrm{~Hz}, 24 \mathrm{H}), 0.88(\mathrm{dd}, J=6.9,2.2 \mathrm{~Hz}, 12 \mathrm{H}) ;{ }^{13} \mathrm{C} \mathrm{NMR}\left(125 \mathrm{MHz}, \mathrm{CDCl}_{3}\right) \delta 171.80,169.87,140.47,139.10$, $134.48,128.69,125.75,124.23,79.61,72.22$, 66.00, 60.71, 58.40, 40.58, 40.39, 37.28, 31.50, 28.57, 28.55, 25.65, 25.59, 22.67, 22.66, 22.30, 14.13; HRMS (ESI): $\mathrm{m} / z$ for $\mathrm{C}_{42} \mathrm{H}_{64} \mathrm{NaO}_{8}[\mathrm{M}+\mathrm{Na}]^{+}$, calcd. 719.4493, found: 719.4490 .

4b: ${ }^{1} \mathrm{H}$ NMR (500 MHz, $\left.\mathrm{CDCl}_{3}\right) \delta 7.07(\mathrm{~d}, J=7.7 \mathrm{~Hz}, 2 \mathrm{H}), 6.98-6.92(\mathrm{~m}, 4 \mathrm{H}), 4.12(\mathrm{t}, J=6.7 \mathrm{~Hz}, 8 \mathrm{H}), 3.99(\mathrm{t}, J=$ $6.7 \mathrm{~Hz}, 4 \mathrm{H}), 3.53(\mathrm{~d}, J=3.1 \mathrm{~Hz}, 8 \mathrm{H}), 3.15(\mathrm{~s}, 4 \mathrm{H}), 1.66-1.58(\mathrm{~m}, 8 \mathrm{H}), 1.54-1.46(\mathrm{~m}, 4 \mathrm{H}), 1.28(\mathrm{~m}, 36 \mathrm{H}), 0.88(\mathrm{~m}$, $18 \mathrm{H}) ;{ }^{13} \mathrm{C}$ NMR $\left(125 \mathrm{MHz}, \mathrm{CDCl}_{3}\right) \delta 171.82,171.20,140.20,138.77,135.24,129.00,126.04,124.01,66.00,65.55$, $60.73,60.52,40.59,40.38,39.19,31.54,31.51,28.58,28.42,25.71,25.60,22.66,14.13$; HRMS (ESI): $\mathrm{m} / \mathrm{z}$ for $\mathrm{C}_{63} \mathrm{H}_{96} \mathrm{NaO}_{12}[\mathrm{M}+\mathrm{Na}]^{+}$, calcd. 1067.6794, found: 1067.6761 .

3d: ${ }^{1} \mathrm{H}$ NMR (500 MHz, $\left.\mathrm{CDCl}_{3}\right) \delta 7.08(\mathrm{~d}, J=7.6 \mathrm{~Hz}, 1 \mathrm{H}), 7.01-6.94(\mathrm{~m}, 2 \mathrm{H}), 4.08(\mathrm{~s}, 4 \mathrm{H}), 3.97$ (dd, $J=31.0$, $11.2 \mathrm{~Hz}, 4 \mathrm{H}), 2.85(\mathrm{~d}, J=5.6 \mathrm{~Hz}, 4 \mathrm{H}), 2.75(\mathrm{~s}, 2 \mathrm{H}), 2.17(\mathrm{~d}, J=2.5 \mathrm{~Hz}, 2 \mathrm{H}), 2.07(\mathrm{~d}, J=14.5 \mathrm{~Hz}, 12 \mathrm{H}) ;{ }^{13} \mathrm{C} \mathrm{NMR}$ $\left(125 \mathrm{MHz}, \mathrm{CDCl}_{3}\right) \delta 171.16,170.76,141.30,139.62,134.27,129.07,126.86,124.91,79.82,72.18,66.87,65.11$, 46.63, 41.04, 38.86, 38.56, 36.81, 21.85, 20.99; HRMS (ESI): $\mathrm{m} / z$ for $\mathrm{C}_{26} \mathrm{H}_{32} \mathrm{NaO}_{8}[\mathrm{M}+\mathrm{Na}]^{+}$, calcd. 495.1989, found: 495.1988 . 
4d: ${ }^{1} \mathrm{H}$ NMR (500 MHz, $\left.\mathrm{CDCl}_{3}\right) \delta 7.06(\mathrm{~d}, J=7.4 \mathrm{~Hz}, 2 \mathrm{H}), 6.87(\mathrm{~d}, J=8.2 \mathrm{~Hz}, 4 \mathrm{H}), 4.08(\mathrm{~s}, 8 \mathrm{H}), 3.83(\mathrm{~s}, 4 \mathrm{H}), 2.84$ (s, 8H), 2.72 (s, 4H), 2.11 (s, 6H), 2.06 (s, 12H); ${ }^{13} \mathrm{C}$ NMR (125 MHz, $\left.\mathrm{CDCl}_{3}\right) \delta 171.18,170.79,141.18,139.39$, 135.17, 129.16, 126.98, 124.82, 66.89, 65.55, 46.62, 41.48, 39.19, 38.85, 38.58, 21.12, 21.01; HRMS (ESI): $\mathrm{m} / \mathrm{z}$ for $\mathrm{C}_{39} \mathrm{H}_{48} \mathrm{NaO}_{12}[\mathrm{M}+\mathrm{Na}]^{+}$, calcd. 731.3038, found: 731.3036 .

3e: ${ }^{1} \mathrm{H}$ NMR $\left(500 \mathrm{MHz}, \mathrm{CDCl}_{3}\right) \delta 7.06(\mathrm{dd}, J=13.0,7.5 \mathrm{~Hz}, 1 \mathrm{H}), 7.02-6.91(\mathrm{~m}, 2 \mathrm{H}), 3.52-3.36(\mathrm{~m}, 6 \mathrm{H}), 3.31-$ $3.10(\mathrm{~m}, 3 \mathrm{H}), 3.07-2.85(\mathrm{~m}, 6 \mathrm{H}), 2.83-2.76(\mathrm{~m}, 1 \mathrm{H}), 2.59-2.52(\mathrm{~m}, 1 \mathrm{H}), 2.40-2.33(\mathrm{~m}, 1 \mathrm{H}), 1.98-1.96(\mathrm{~m}$, $1 \mathrm{H}), 1.22(\mathrm{td}, J=7.1,1.4 \mathrm{~Hz}, 3 \mathrm{H}), 1.13(\mathrm{t}, J=7.1 \mathrm{~Hz}, 3 \mathrm{H}), 1.02(\mathrm{q}, J=7.2 \mathrm{~Hz}, 3 \mathrm{H}), 0.94(\mathrm{dt}, J=11.0,7.2 \mathrm{~Hz}, 3 \mathrm{H})$; ${ }^{13} \mathrm{C} \mathrm{NMR}\left(75 \mathrm{MHz}, \mathrm{CDCl}_{3}\right) \delta 173.87,173.00,142.37,140.33,137.52,127.60,125.16,124.14,82.57,69.73,63.98$, 43.42, 42.07, 40.48, 39.09, 37.17, 22.47, 15.03, 14.65, 13.22; HRMS (ESI): $m / z$ for $\mathrm{C}_{24} \mathrm{H}_{34} \mathrm{~N}_{2} \mathrm{NaO}_{2}[\mathrm{M}+\mathrm{Na}]^{+}$, calcd. 405.2512, found: 405.2513 .

4e: ${ }^{1} \mathrm{H}$ NMR (500 MHz, $\left.\mathrm{CDCl}_{3}\right) \delta 7.05(\mathrm{t}, J=7.7 \mathrm{~Hz}, 2 \mathrm{H}), 6.99(\mathrm{~s}, 2 \mathrm{H}), 6.96-6.92(\mathrm{~m}, 2 \mathrm{H}), 3.51-3.36(\mathrm{~m}, 10 \mathrm{H})$, $3.31-3.16(\mathrm{~m}, 6 \mathrm{H}), 3.06-2.87(\mathrm{~m}, 7 \mathrm{H}), 2.74-2.61(\mathrm{~m}, 4 \mathrm{H}), 1.24-1.20(\mathrm{~m}, 6 \mathrm{H}), 1.14(\mathrm{t}, J=7.1 \mathrm{~Hz}, 6 \mathrm{H}), 0.97-$ $0.90(\mathrm{~m}, 3 \mathrm{H}), 0.62-0.55$ (m, 3H); ${ }^{13} \mathrm{C}$ NMR (126 MHz, $\left.\mathrm{cdcl}_{3}\right) \delta 173.87,142.14,139.93,138.39,127.38,125.11$, 123.96, 46.88, 42.02, 41.48, 40.44, 39.58, 37.03, 15.01, 14.10, 13.20; HRMS (ESI): $\mathrm{m} / z$ for $\mathrm{C}_{36} \mathrm{H}_{51} \mathrm{~N}_{3} \mathrm{NaO}_{3}[\mathrm{M}+\mathrm{Na}]^{+}$, calcd. 596.3823, found: 596.3822 .

3f: ${ }^{1} \mathrm{H}$ NMR $\left(500 \mathrm{MHz}, \mathrm{CDCl}_{3}\right) \delta 7.11(\mathrm{~d}, J=7.6 \mathrm{~Hz}, 1 \mathrm{H}), 7.03(\mathrm{~s}, 1 \mathrm{H}), 6.97(\mathrm{~d}, J=7.6 \mathrm{~Hz}, 1 \mathrm{H}), 4.14-4.01(\mathrm{~m}$, 4H), $3.36-3.27(\mathrm{~m}, 1 \mathrm{H}), 3.26-3.11(\mathrm{~m}, 4 \mathrm{H}), 3.03-2.85(\mathrm{~m}, 2 \mathrm{H}), 2.86-2.78(\mathrm{~m}, 1 \mathrm{H}), 2.48-2.36(\mathrm{~m}, 2 \mathrm{H}), 2.02(\mathrm{t}$, $J=2.5 \mathrm{~Hz}, 1 \mathrm{H}), 1.68-1.52(\mathrm{~m}, 4 \mathrm{H}), 1.26(\mathrm{~s}, 28 \mathrm{H}), 0.88(\mathrm{t}, J=6.6 \mathrm{~Hz}, 6 \mathrm{H}) ;{ }^{13} \mathrm{C} \mathrm{NMR}\left(125 \mathrm{MHz}, \mathrm{CDCl}_{3}\right) \delta 175.45$, 173.90, 142.15, 140.10, 137.02, 127.56, 125.09, 124.39, 81.47, 70.38, 65.02, 46.52, 44.02, 36.83, 36.26, 36.04, 32.05, 29.69, 29.68, 29.67, 29.46, 29.40, 29.38, 28.81, 28.73, 26.07, 26.01, 22.83, 20.60, 14.26; HRMS (ESI): $\mathrm{m} / \mathrm{z}$ for $\mathrm{C}_{36} \mathrm{H}_{56} \mathrm{NaO}_{4}[\mathrm{M}+\mathrm{Na}]^{+}$, calcd. 575.4071, found: 575.4073 .

4f: ${ }^{1} \mathrm{H}$ NMR (500 MHz, $\left.\mathrm{CDCl}_{3}\right) \delta 7.09(\mathrm{~d}, J=7.6 \mathrm{~Hz}, 2 \mathrm{H}), 6.99(\mathrm{~s}, 2 \mathrm{H}), 6.94(\mathrm{~d}, J=7.6 \mathrm{~Hz}, 2 \mathrm{H}), 4.11(\mathrm{t}, J=6.7 \mathrm{~Hz}$, $4 \mathrm{H}), 3.90(\mathrm{t}, J=6.6 \mathrm{~Hz}, 2 \mathrm{H}), 3.35-3.26(\mathrm{~m}, 2 \mathrm{H}), 3.25-3.10(\mathrm{~m}, 8 \mathrm{H}), 2.95-2.87(\mathrm{~m}, 3 \mathrm{H}), 2.74(\mathrm{~d}, J=8.6 \mathrm{~Hz}, 2 \mathrm{H})$, $1.68-1.60(\mathrm{~m}, 4 \mathrm{H}), 1.44-1.07(\mathrm{~m}, 44 \mathrm{H}), 0.88(\mathrm{t}, J=6.9 \mathrm{~Hz}, 9 \mathrm{H}) ;{ }^{13} \mathrm{C} \mathrm{NMR}\left(125 \mathrm{MHz}, \mathrm{CDCl}_{3}\right) \delta$ 175.47, 175.20, 142.01, 139.81, 137.76, 127.44, 124.95, 124.27, 64.98, 64.55, 50.07, 44.03, 38.26, 36.25, 36.02, 32.04, 29.71, 29.67, 29.47, 29.45, 29.40, 28.80, 28.64, 26.06, 25.93, 22.83, 14.27; HRMS (ESI): $m / z$ for $\mathrm{C}_{54} \mathrm{H}_{84} \mathrm{NaO}_{6}[\mathrm{M}+\mathrm{Na}]^{+}$, calcd. 851.6160 , found: 851.6158 . 
Ring structure of the polymer synthesized by HG2: ${ }^{13} \mathrm{C}$ NMR spectroscopy

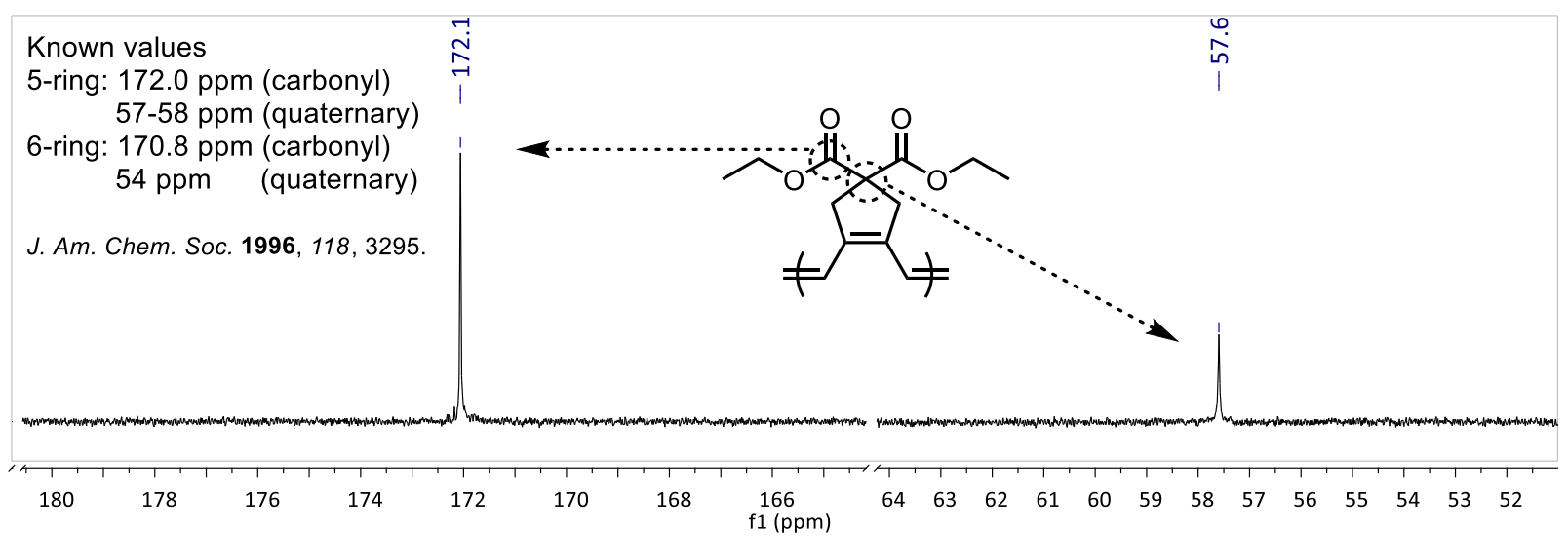

\section{${ }^{1} \mathrm{H}$ and ${ }^{13} \mathrm{C}$ NMR spectra}

1e $\left({ }^{1} \mathrm{H} \mathrm{NMR}, \mathrm{CDCl}_{3}\right)$

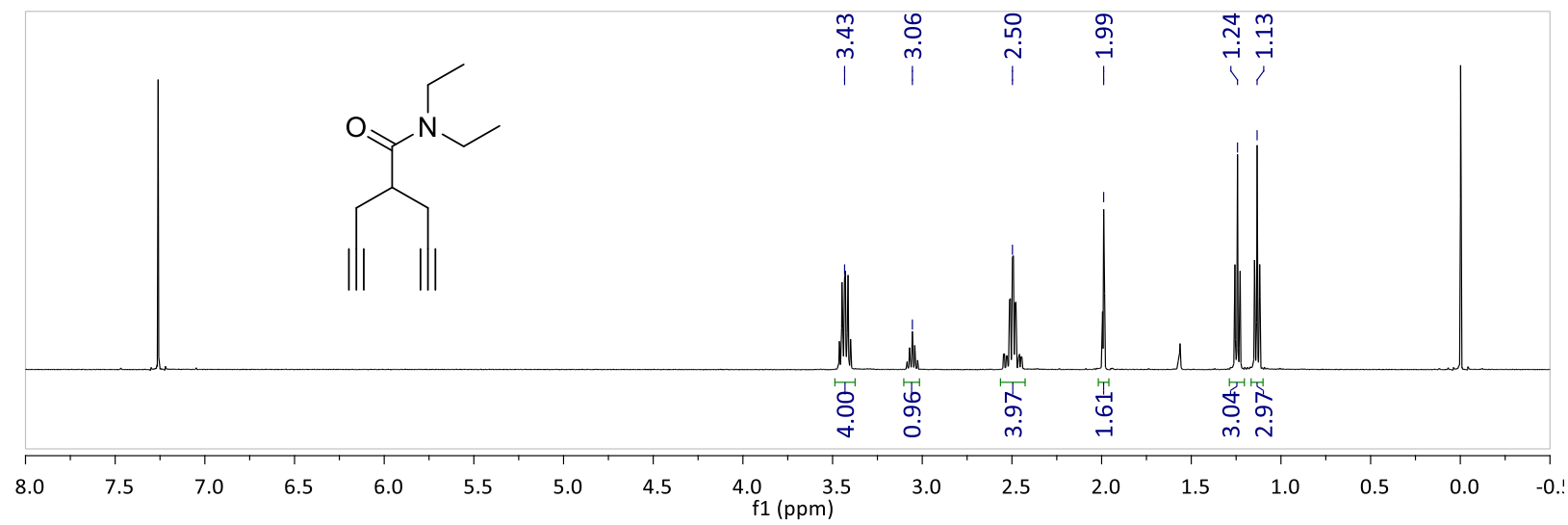

1e $\left({ }^{13} \mathrm{C} \mathrm{NMR}, \mathrm{CDCl}_{3}\right)$

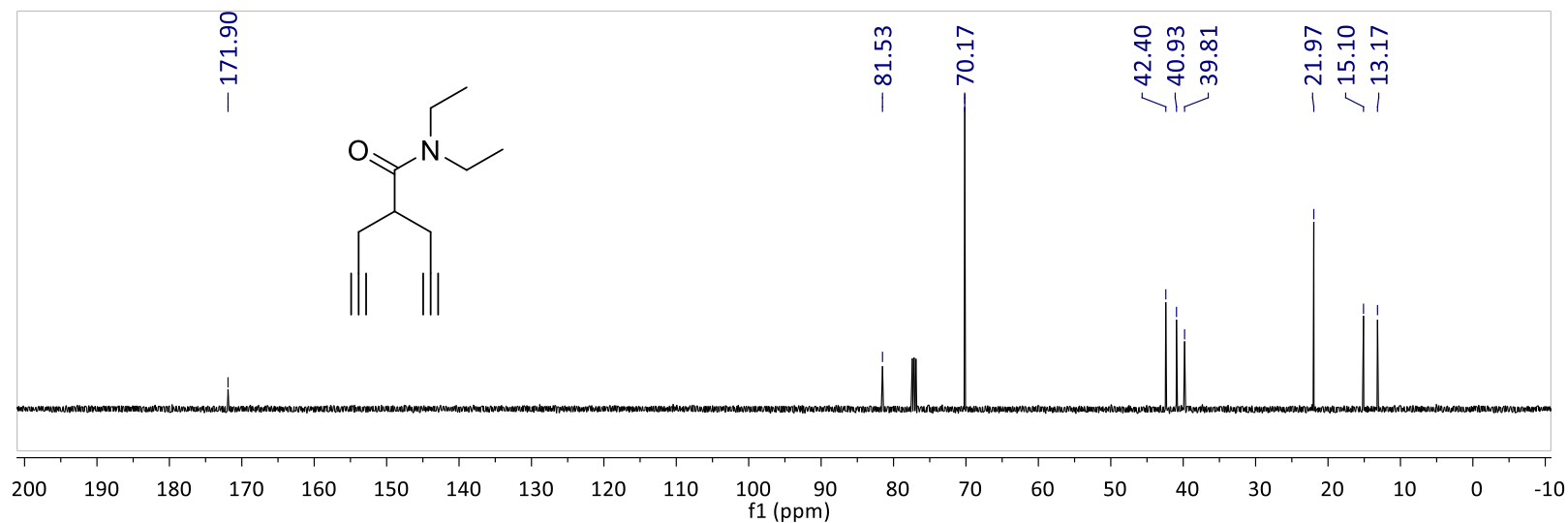


1f $\left({ }^{1} \mathrm{H} \mathrm{NMR}, \mathrm{CDCl}_{3}\right)$

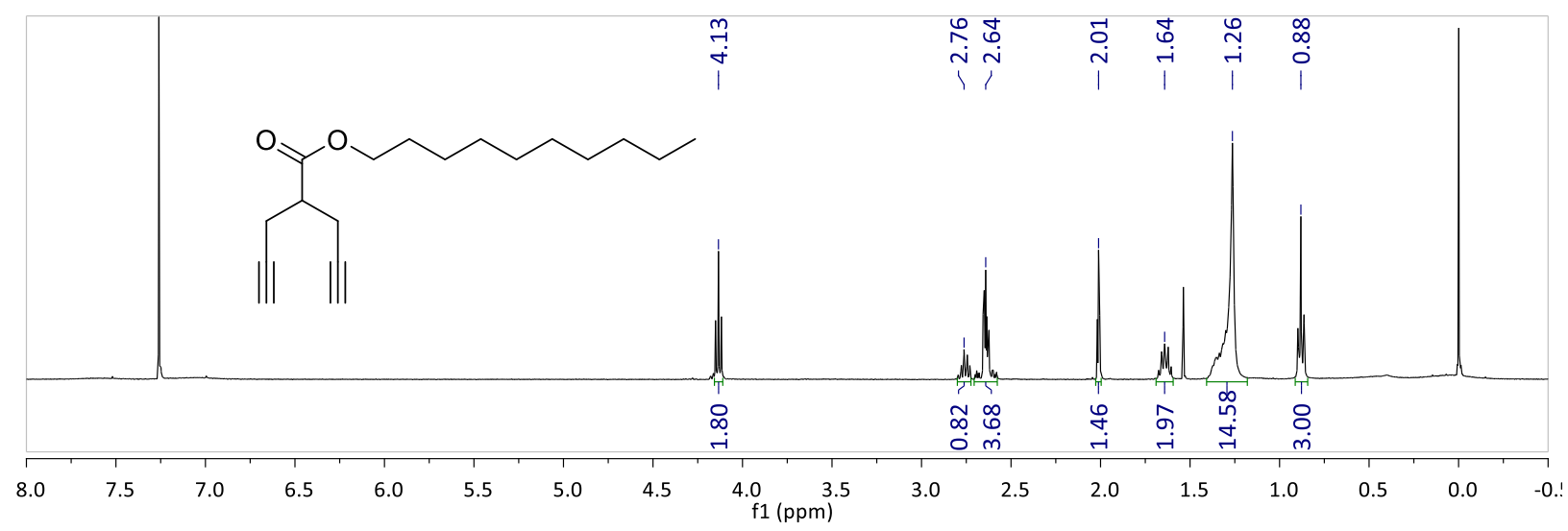

If $\left({ }^{13} \mathrm{C} \mathrm{NMR}, \mathrm{CDCl}_{3}\right)$

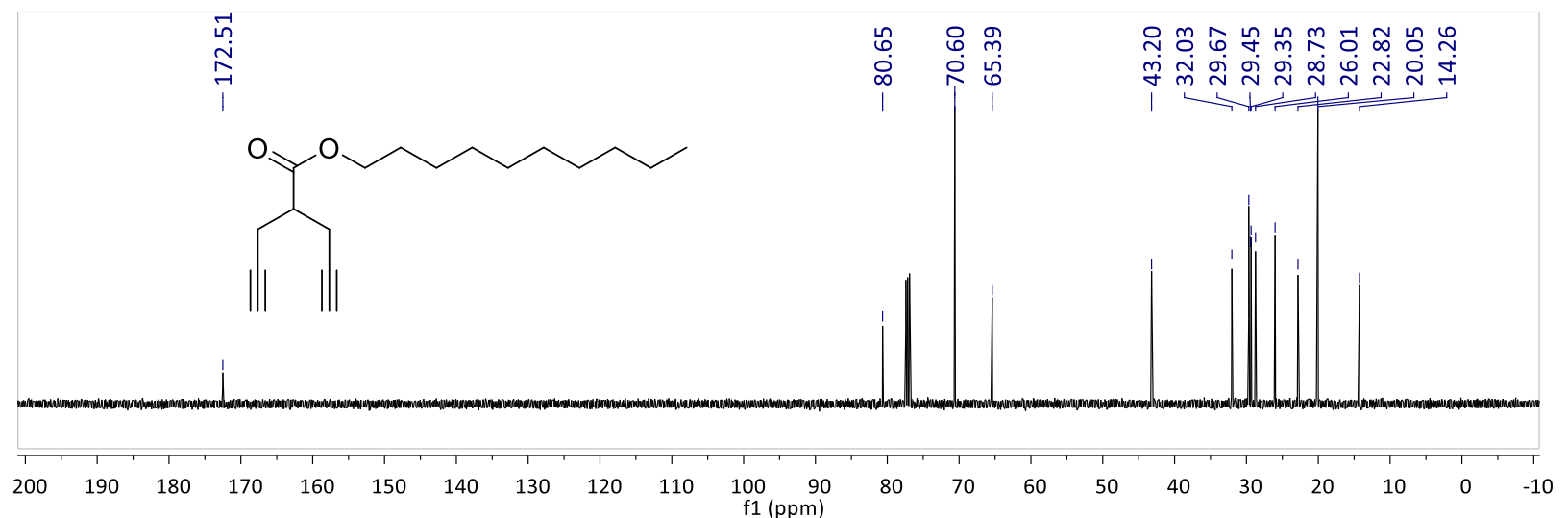

1h $\left({ }^{1} \mathrm{H} \mathrm{NMR}, \mathrm{CDCl}_{3}\right)$

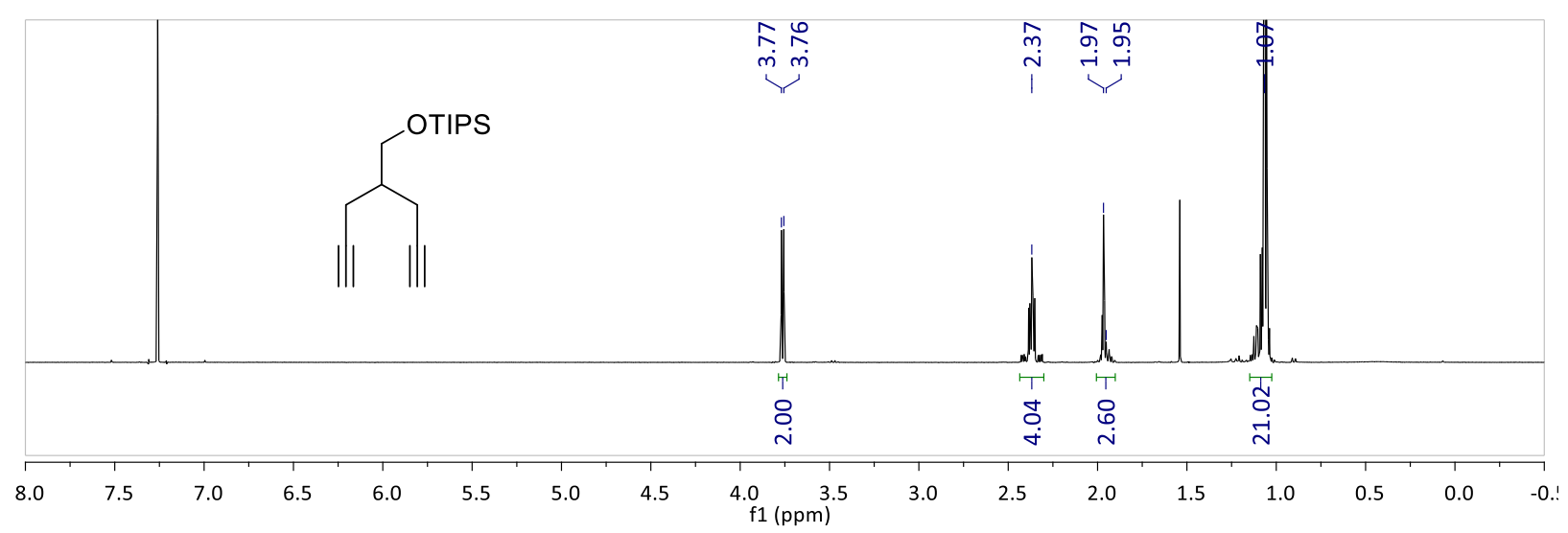


1h $\left({ }^{13} \mathrm{C} \mathrm{NMR}, \mathrm{CDCl}_{3}\right)$

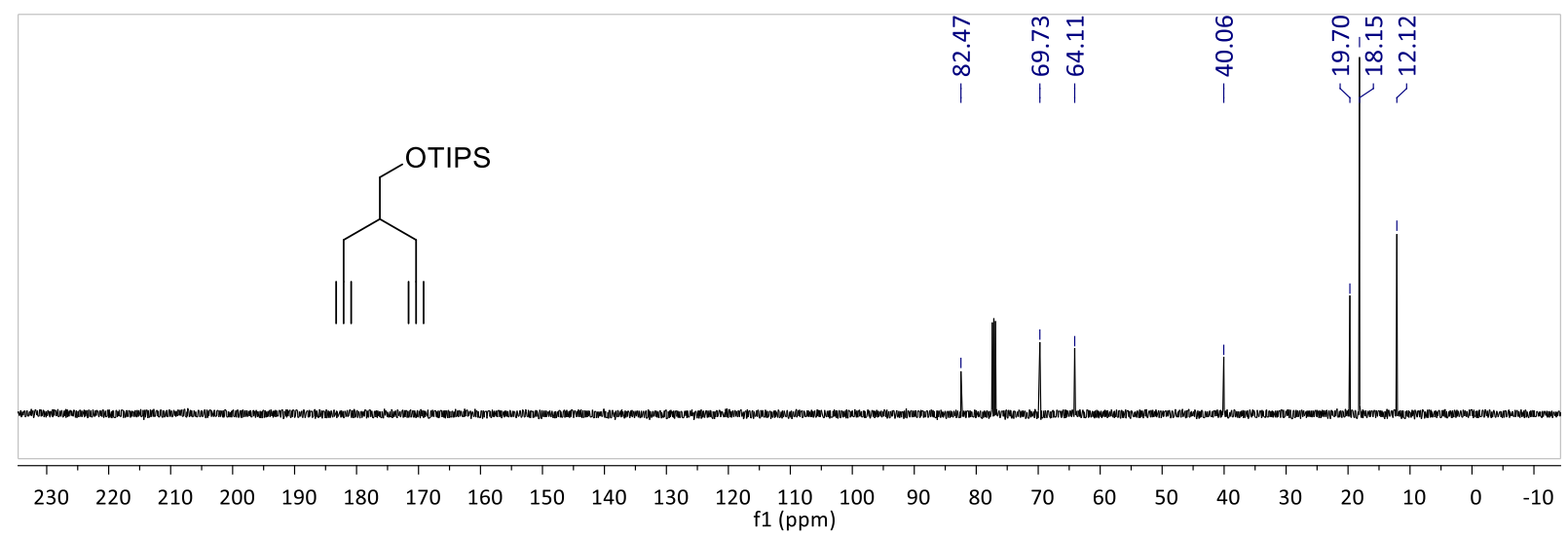

\section{2e $\left({ }^{1} \mathrm{H}\right.$ NMR, $\left.\mathrm{CD}_{2} \mathrm{Cl}_{2}\right)$}

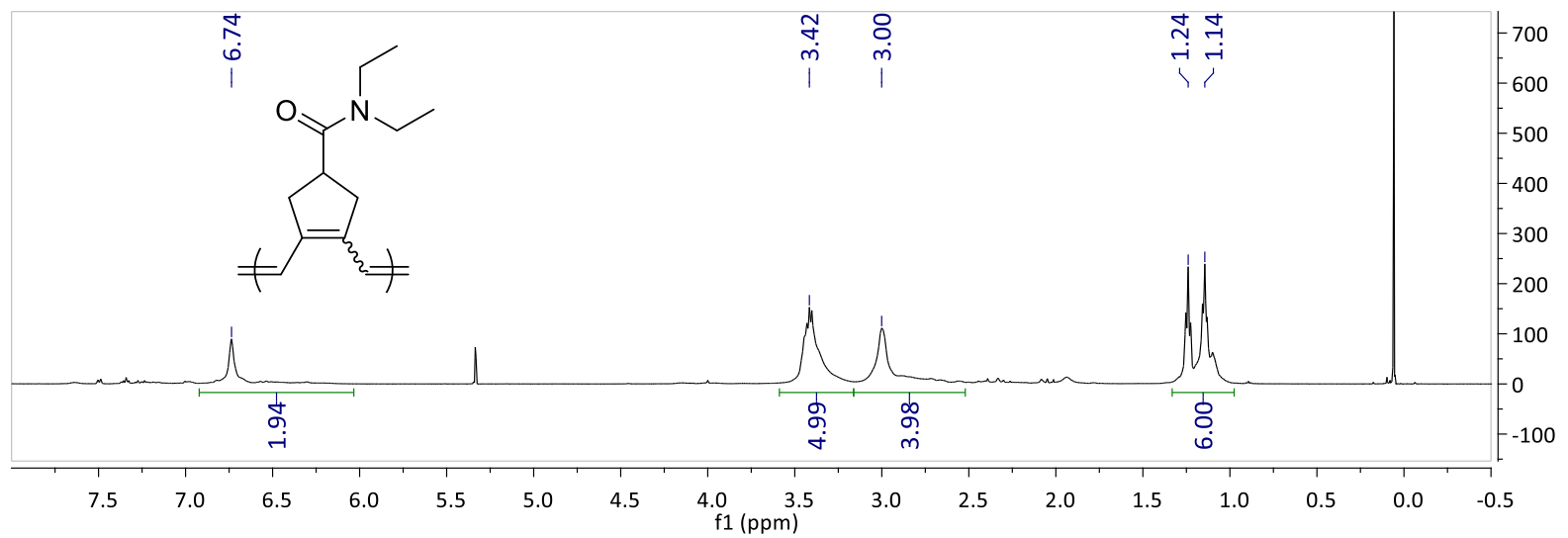

2e $\left({ }^{13} \mathrm{C} \mathrm{NMR}, \mathrm{CD}_{2} \mathrm{Cl}_{2}\right)$

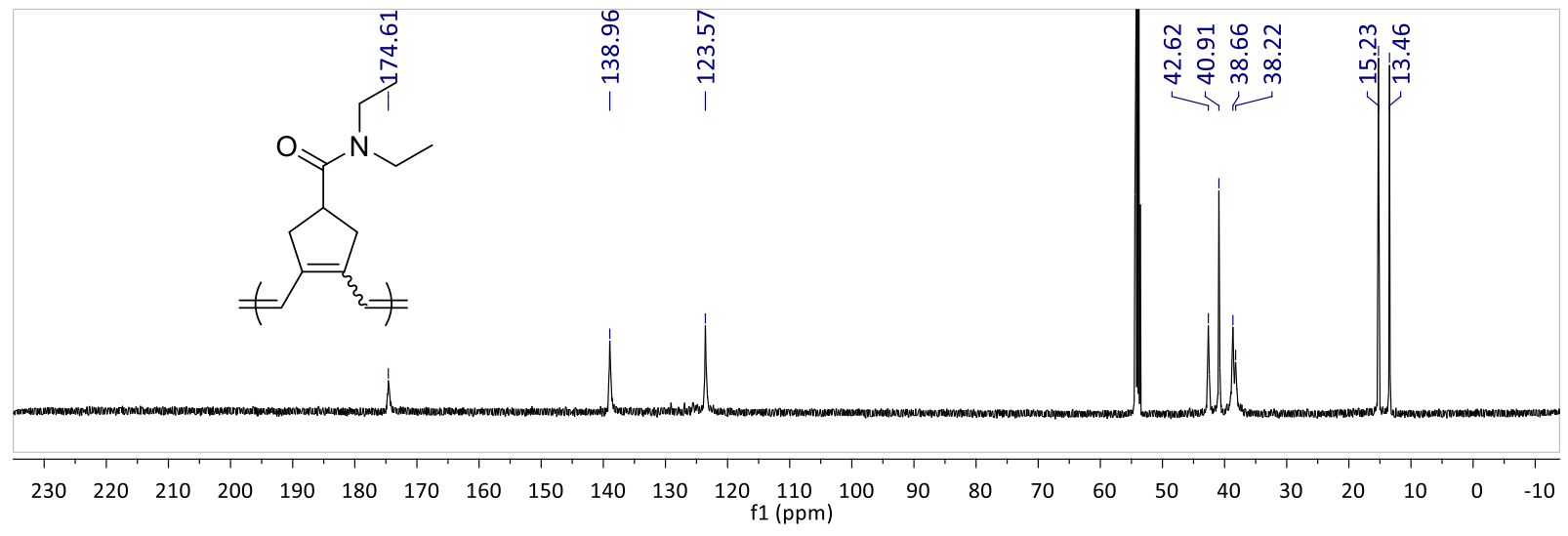


2f $\left({ }^{1} \mathrm{H} \mathrm{NMR}, \mathrm{CDCl}_{3}\right)$

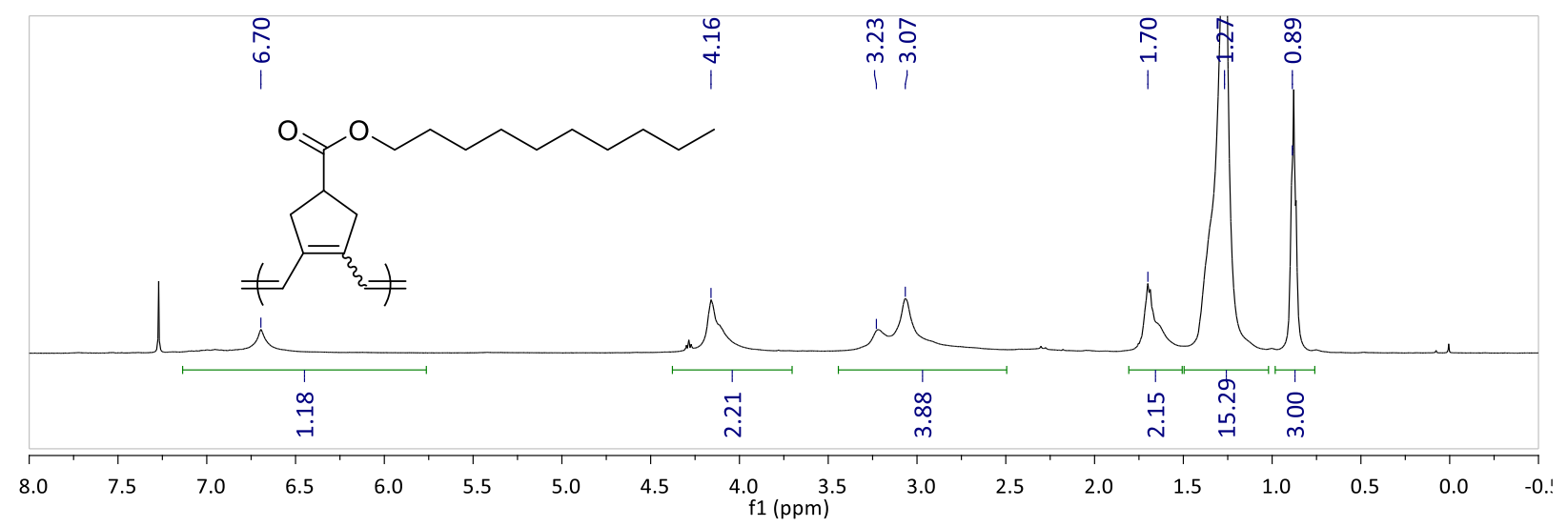

2f $\left({ }^{13} \mathrm{C} \mathrm{NMR}, \mathrm{CDCl}_{3}\right)$

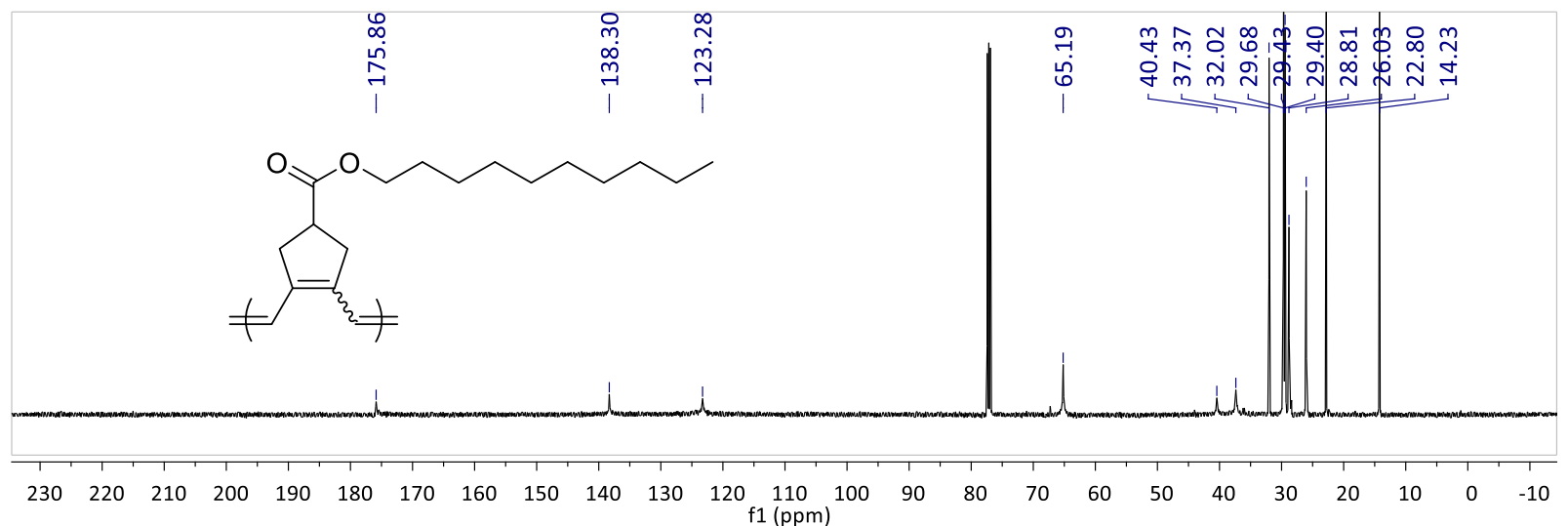

\section{2h $\left({ }^{1} \mathrm{H} \mathrm{NMR}, \mathrm{CDCl}_{3}\right)$}

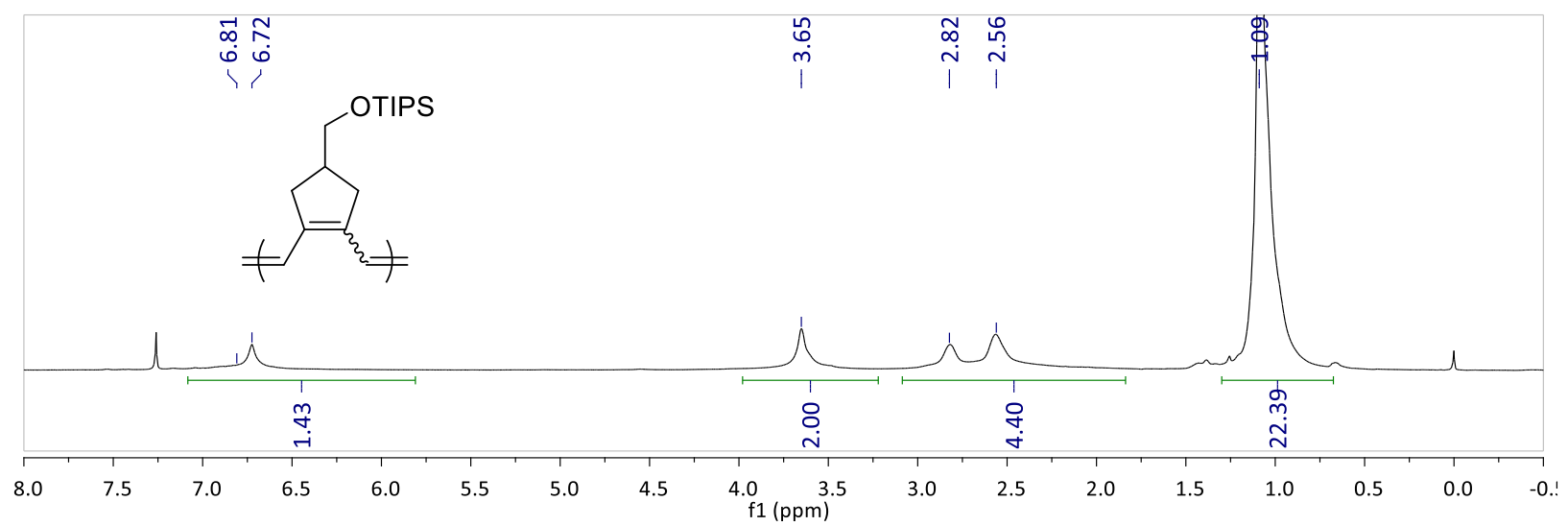


2h $\left({ }^{13} \mathrm{C} \mathrm{NMR}, \mathrm{CDCl}_{3}\right)$

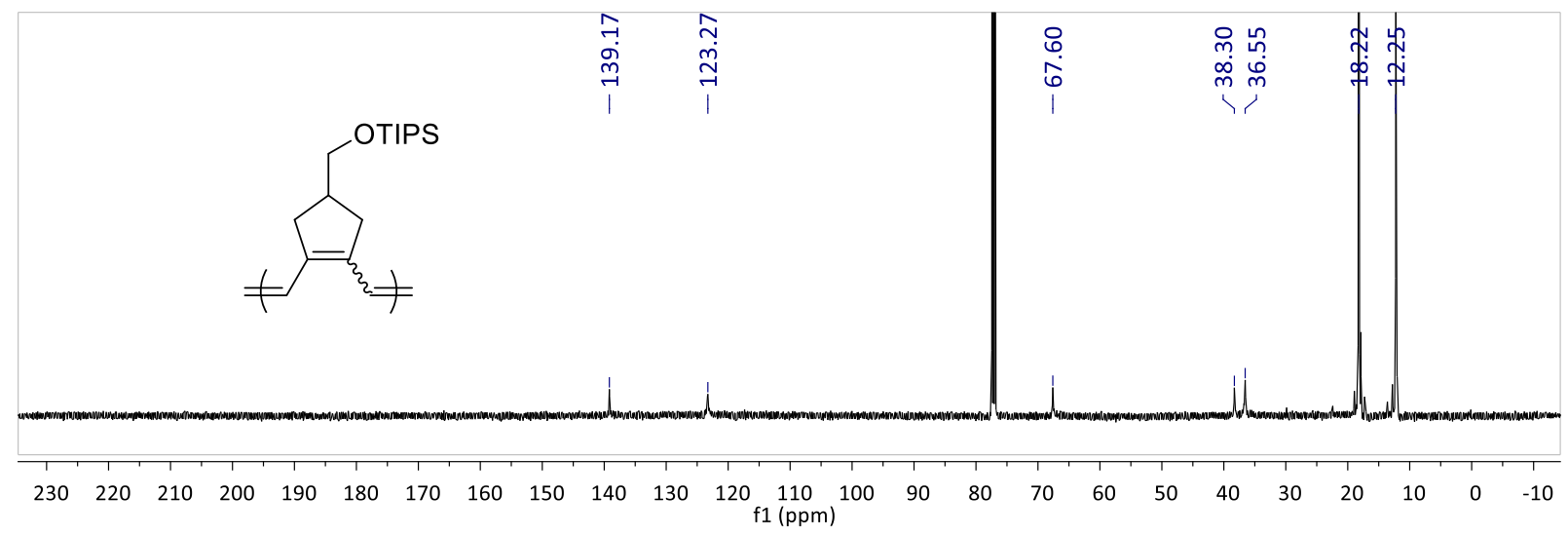

4a $\left({ }^{1} \mathrm{H}\right.$ NMR, $\left.\mathrm{CDCl}_{3}\right)$

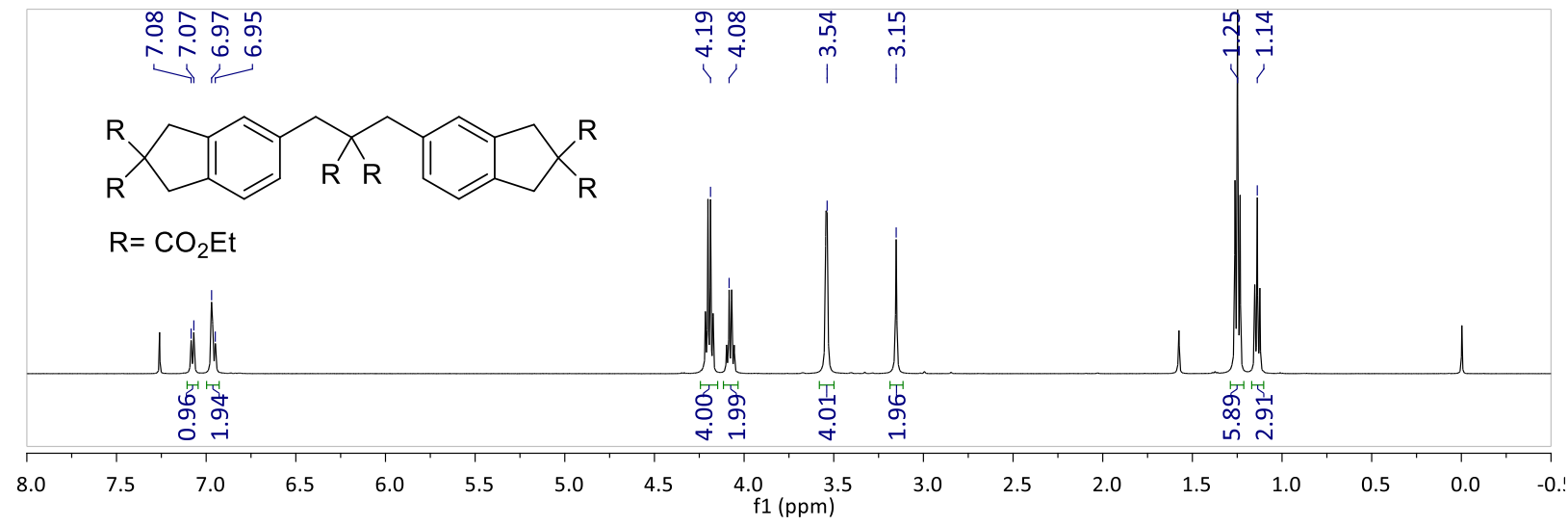

4a $\left({ }^{13} \mathrm{C} \mathrm{NMR}, \mathrm{CDCl}_{3}\right)$

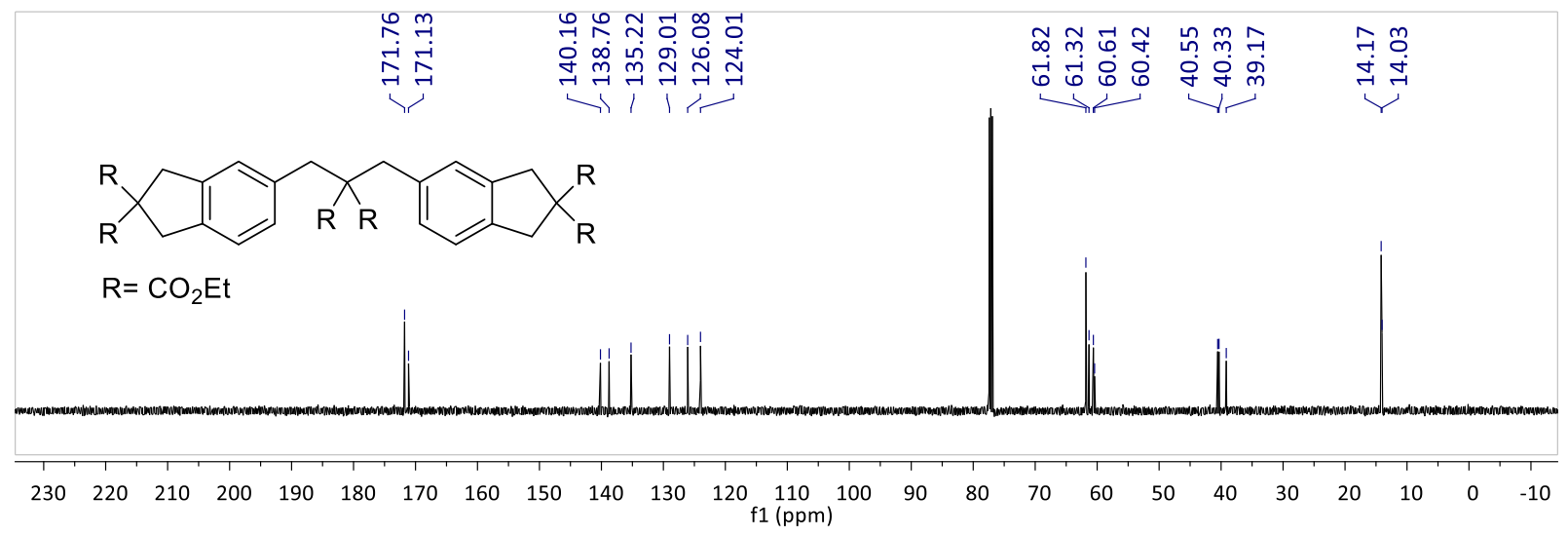




\section{3b $\left({ }^{1} \mathrm{H}\right.$ NMR, $\left.\mathrm{CDCl}_{3}\right)$}

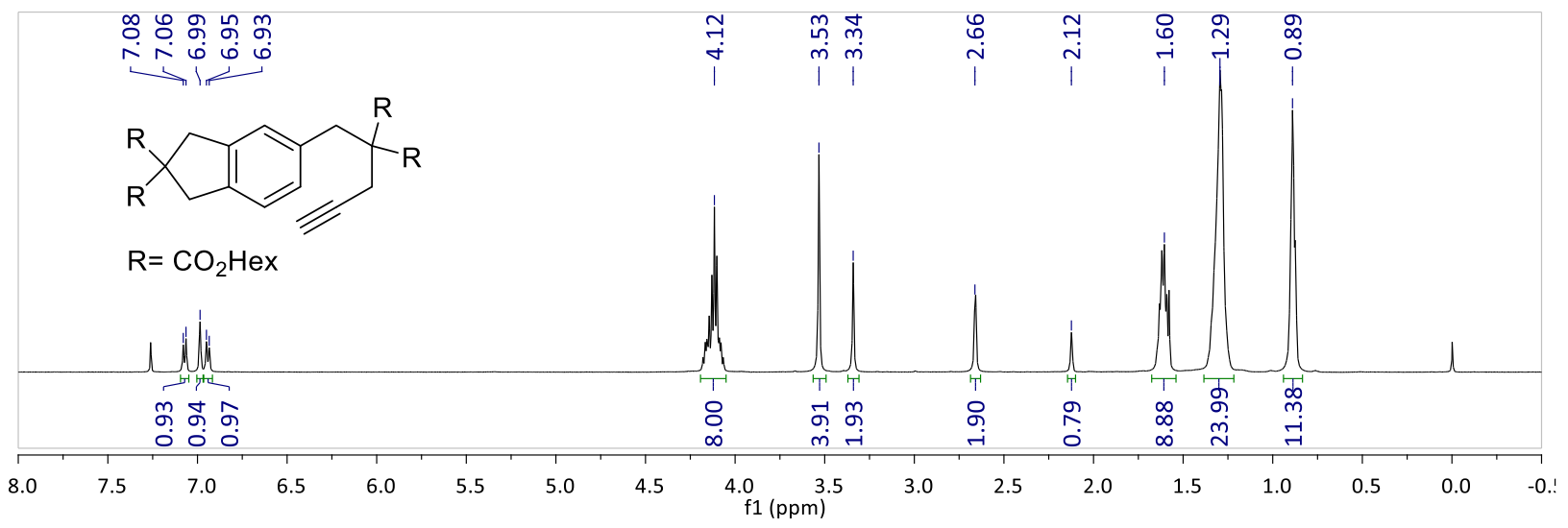

3b $\left({ }^{13} \mathrm{C} \mathrm{NMR}, \mathrm{CDCl}_{3}\right)$

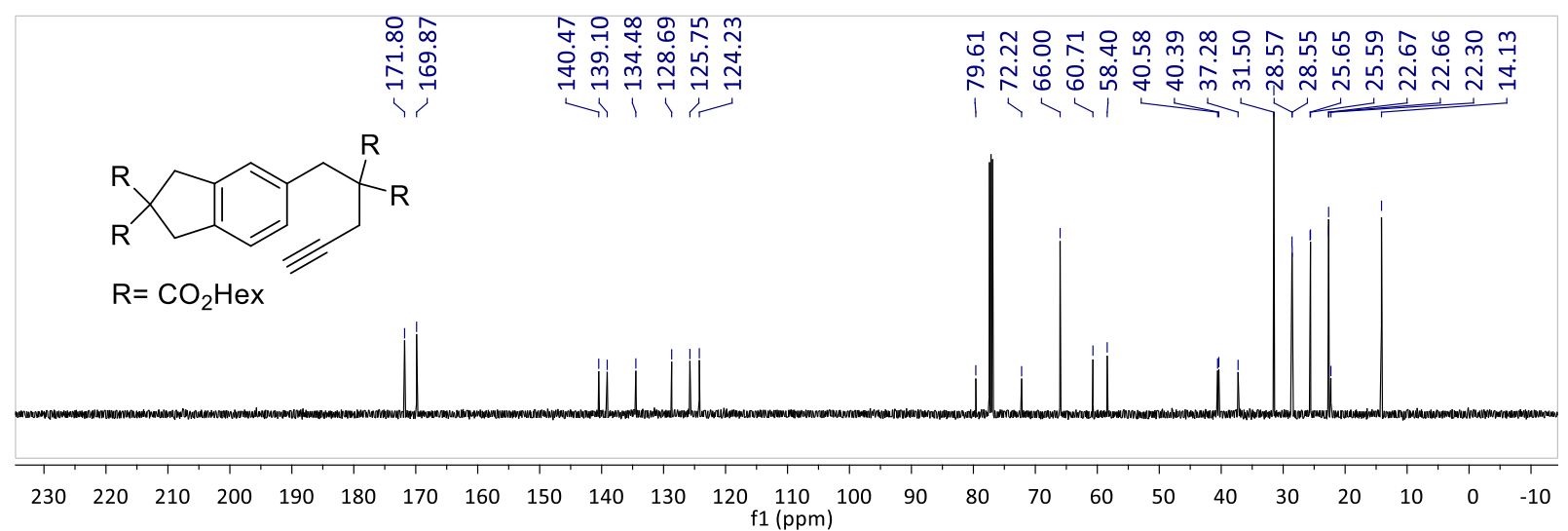

4b $\left({ }^{1} \mathrm{H} \mathrm{NMR}, \mathrm{CDCl}_{3}\right)$

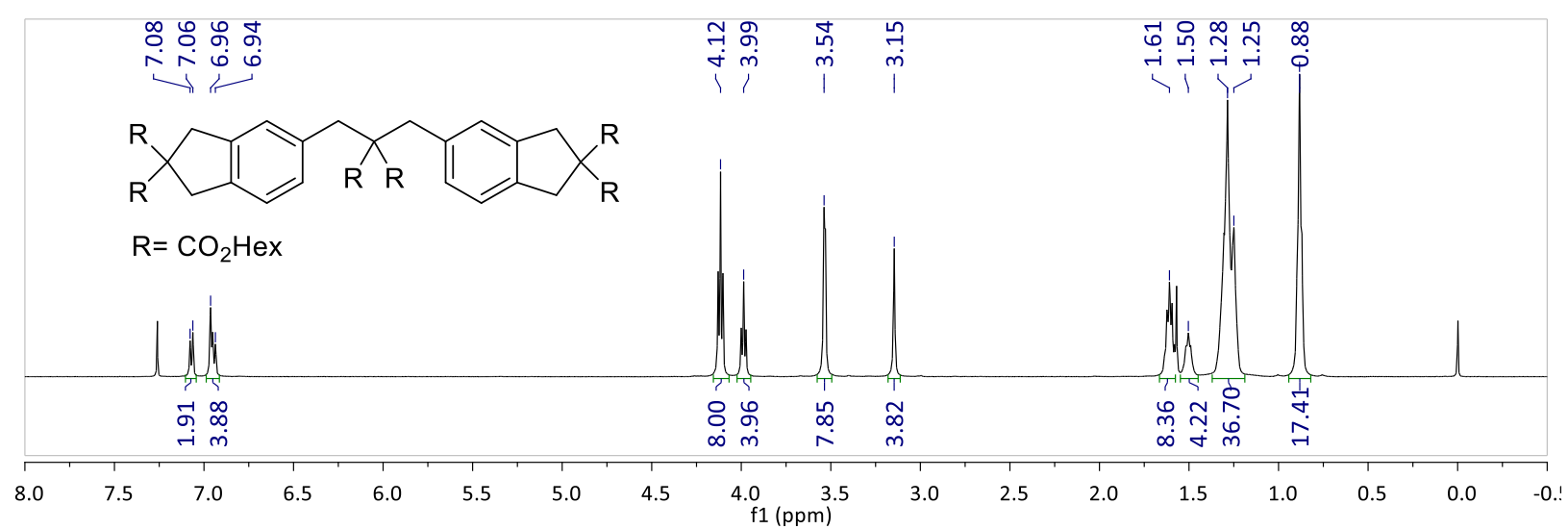


4b $\left({ }^{13} \mathrm{C} \mathrm{NMR}, \mathrm{CDCl}_{3}\right)$

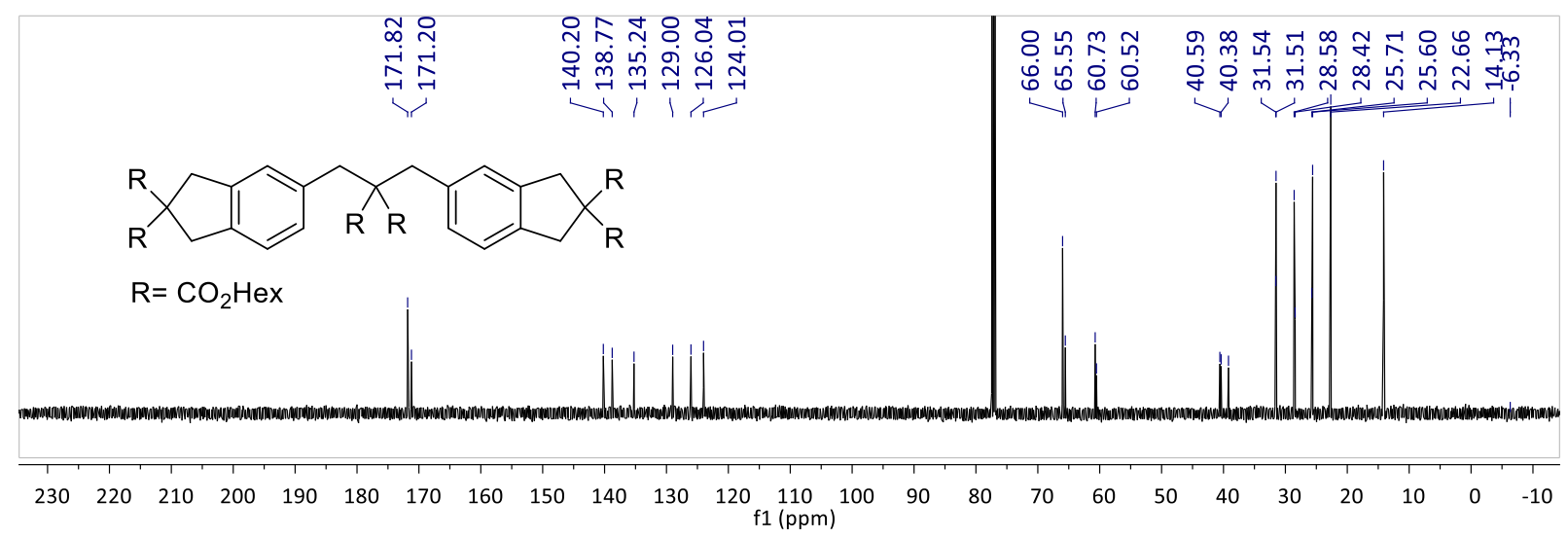

3d $\left({ }^{1} \mathrm{H} \mathrm{NMR}, \mathrm{CDCl}_{3}\right)$

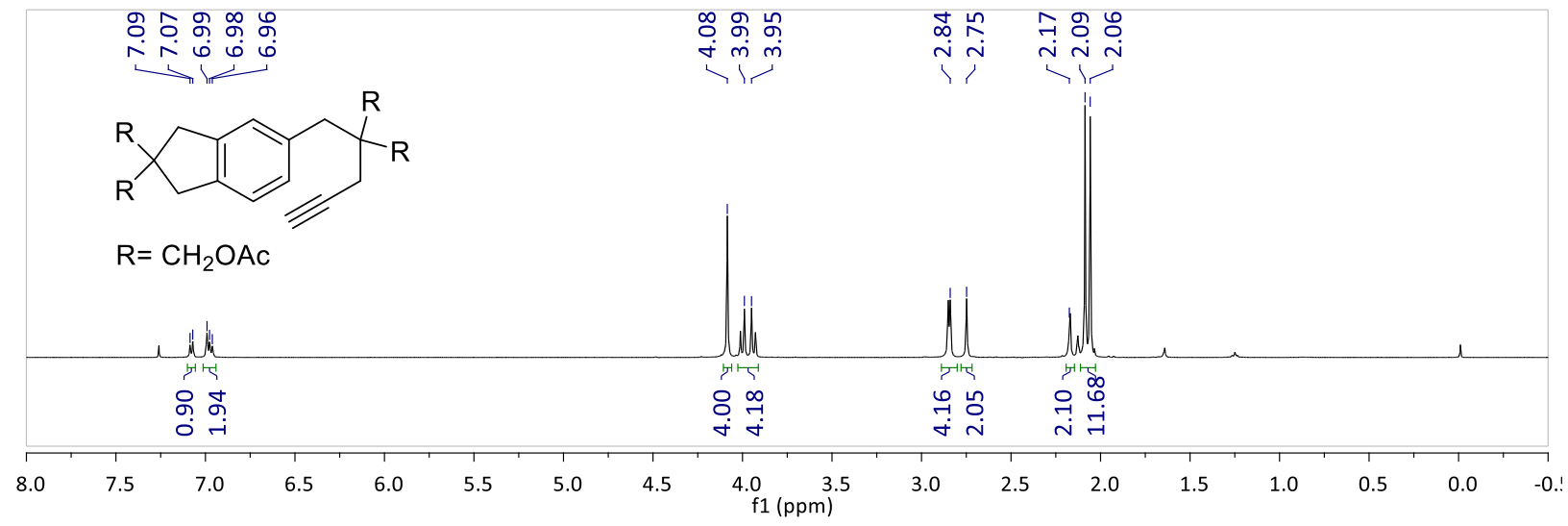

\section{3d $\left({ }^{13} \mathrm{C} \mathrm{NMR}, \mathrm{CDCl}_{3}\right)$}

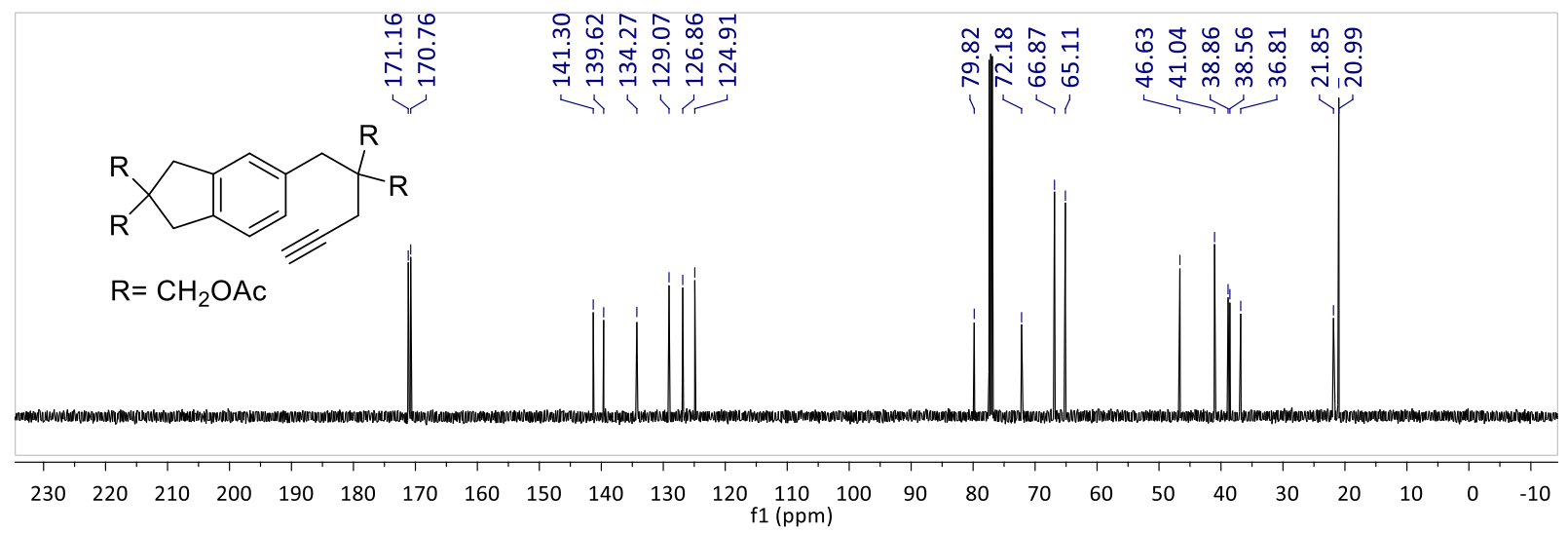


4d ( $\left.{ }^{1} \mathrm{H} \mathrm{NMR}, \mathrm{CDCl}_{3}\right)$

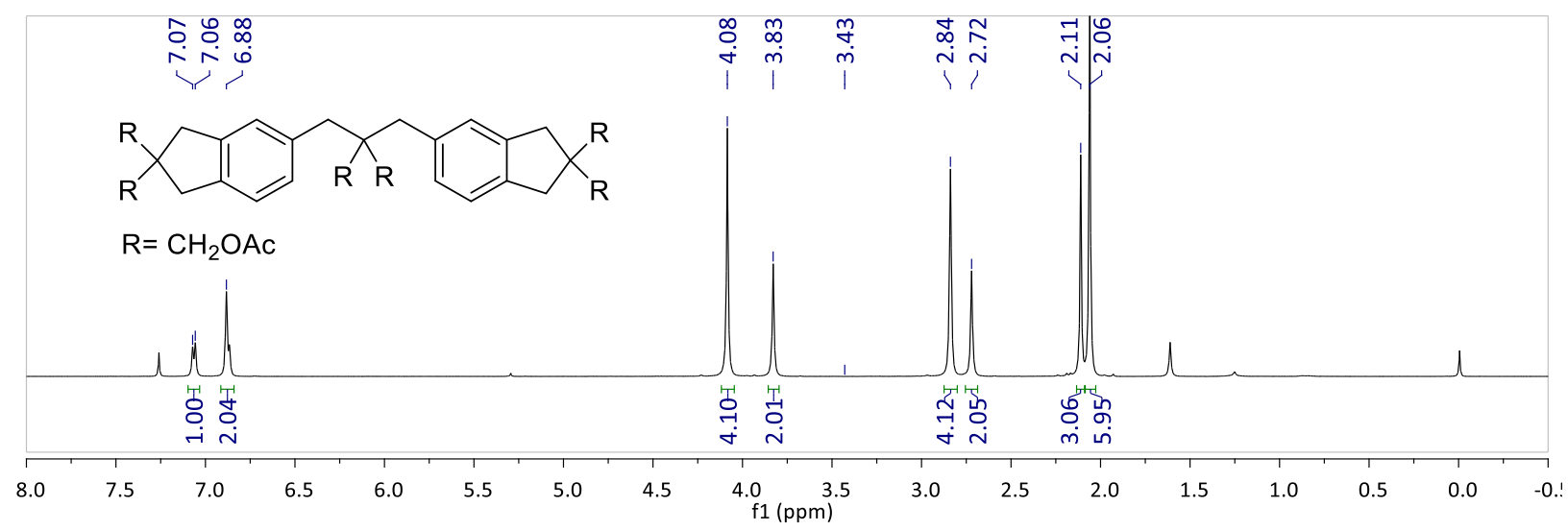

4d $\left({ }^{13} \mathrm{C} \mathrm{NMR,} \mathrm{CDCl}_{3}\right)$

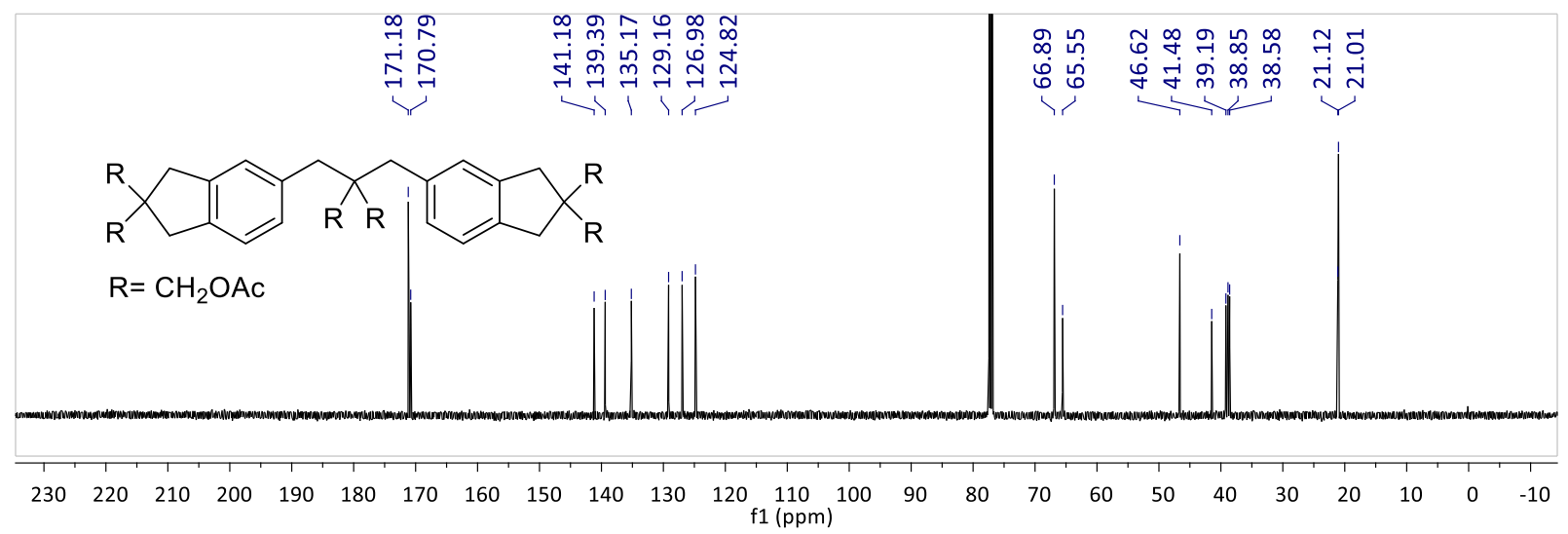

3e $\left({ }^{1} \mathrm{H} \mathrm{NMR}, \mathrm{CDCl}_{3}\right)$

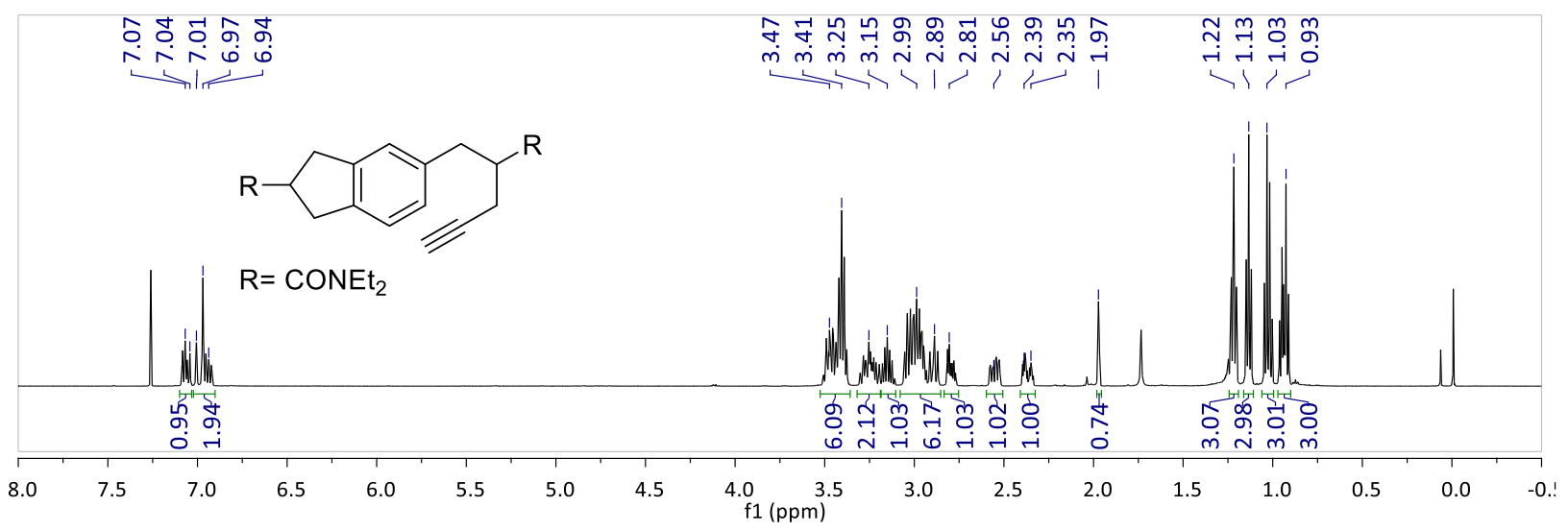


3e $\left({ }^{13} \mathrm{C} \mathrm{NMR}, \mathrm{CDCl}_{3}\right)$

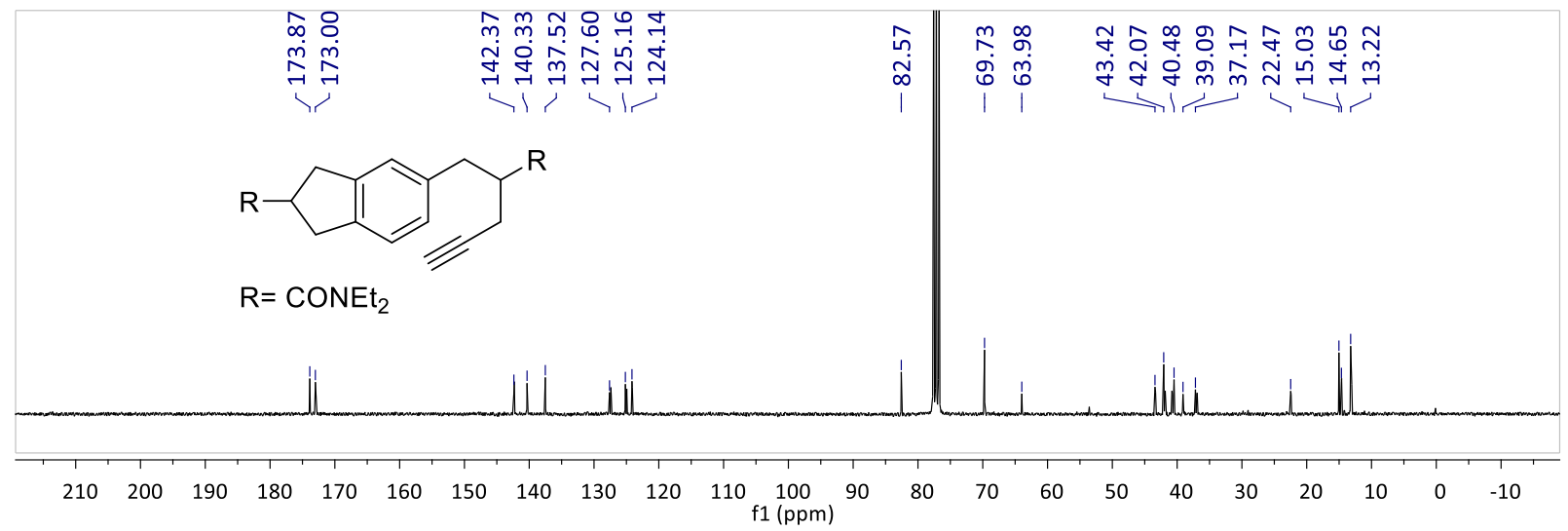

4e ( ${ }^{1} \mathrm{H}$ NMR, $\left.\mathrm{CDCl}_{3}\right)$

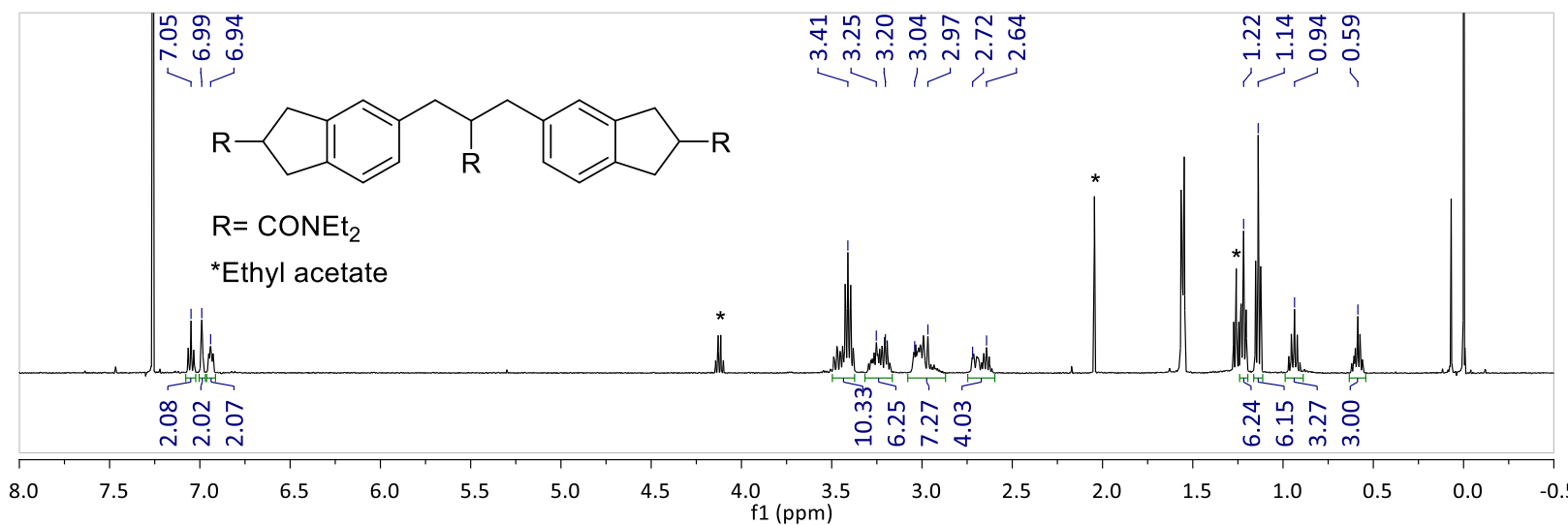

4e $\left({ }^{13} \mathrm{C} \mathrm{NMR}, \mathrm{CDCl}_{3}\right)$

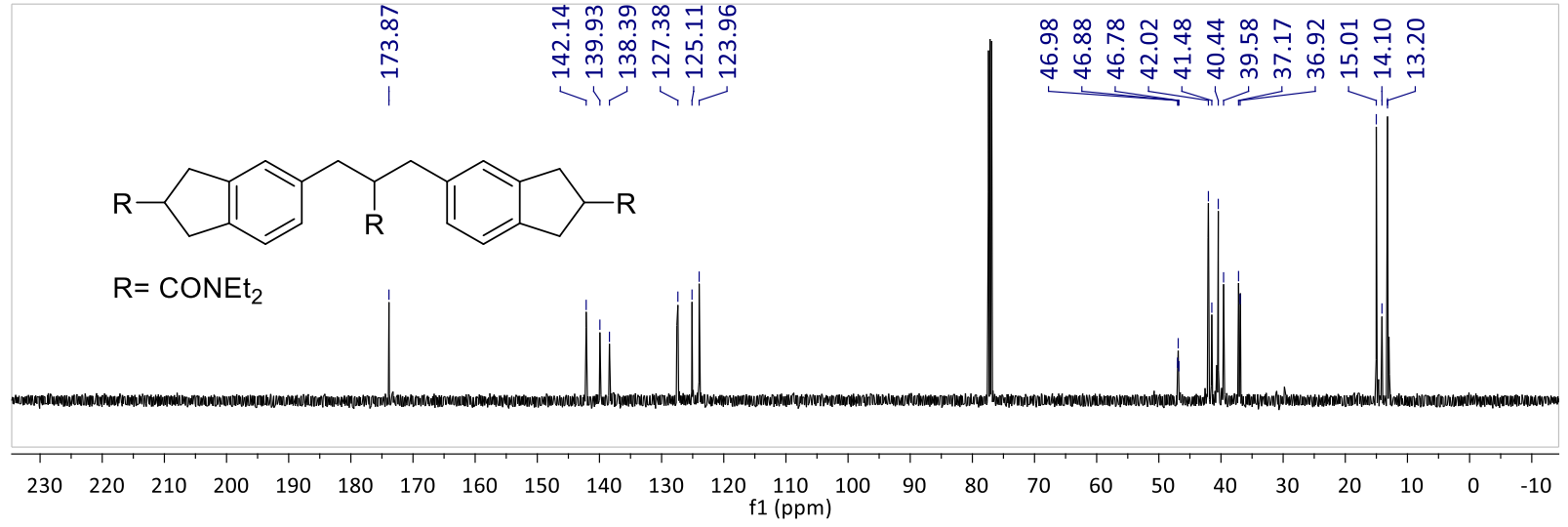


3f $\left({ }^{1} \mathrm{H}\right.$ NMR, $\left.\mathrm{CDCl}_{3}\right)$

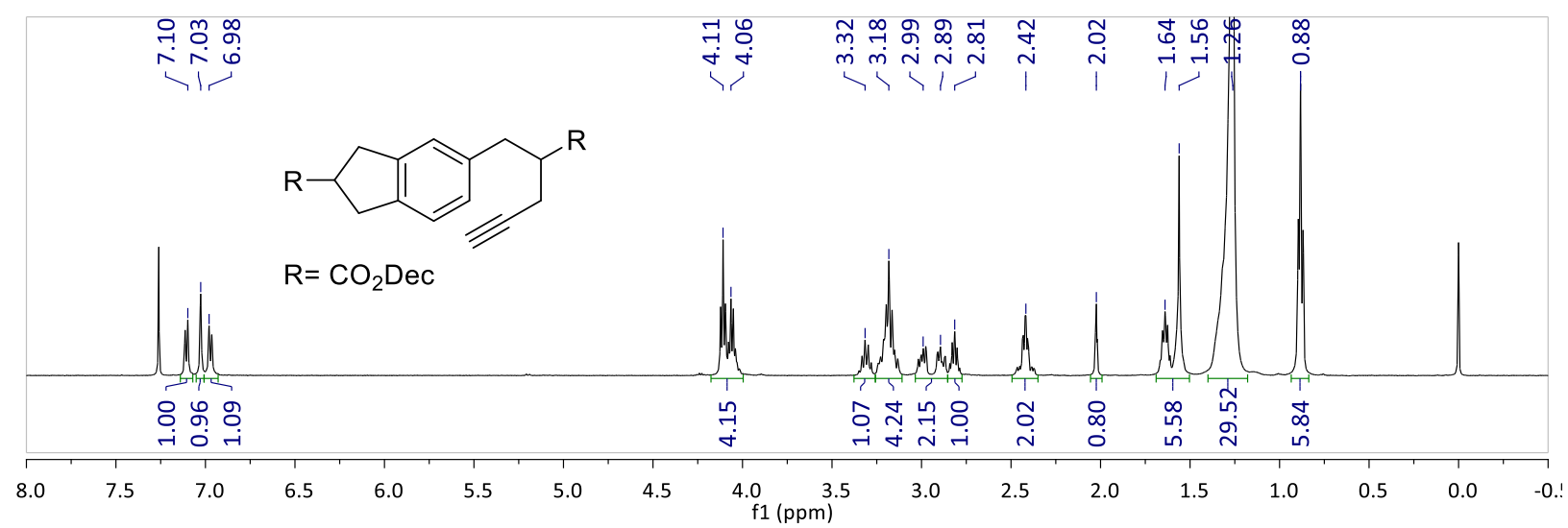

\section{3f $\left({ }^{13} \mathrm{C} \mathrm{NMR}, \mathrm{CDCl}_{3}\right)$}

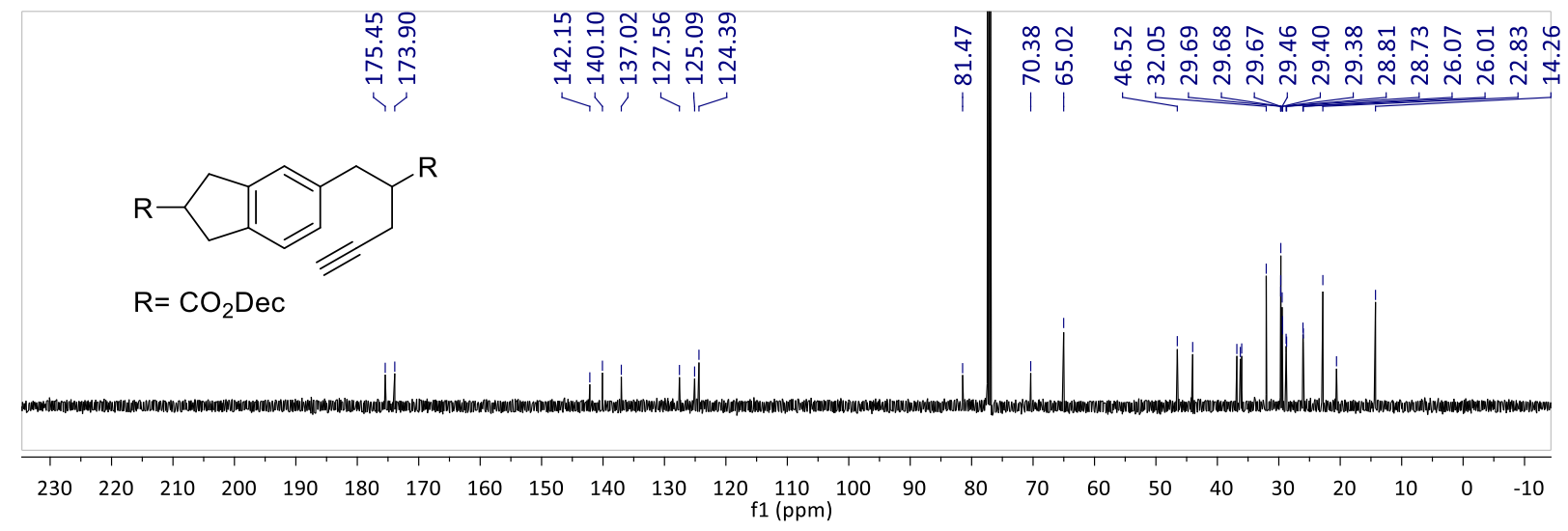

\section{4f $\left({ }^{1} \mathrm{H} \mathrm{NMR}, \mathrm{CDCl}_{3}\right)$}

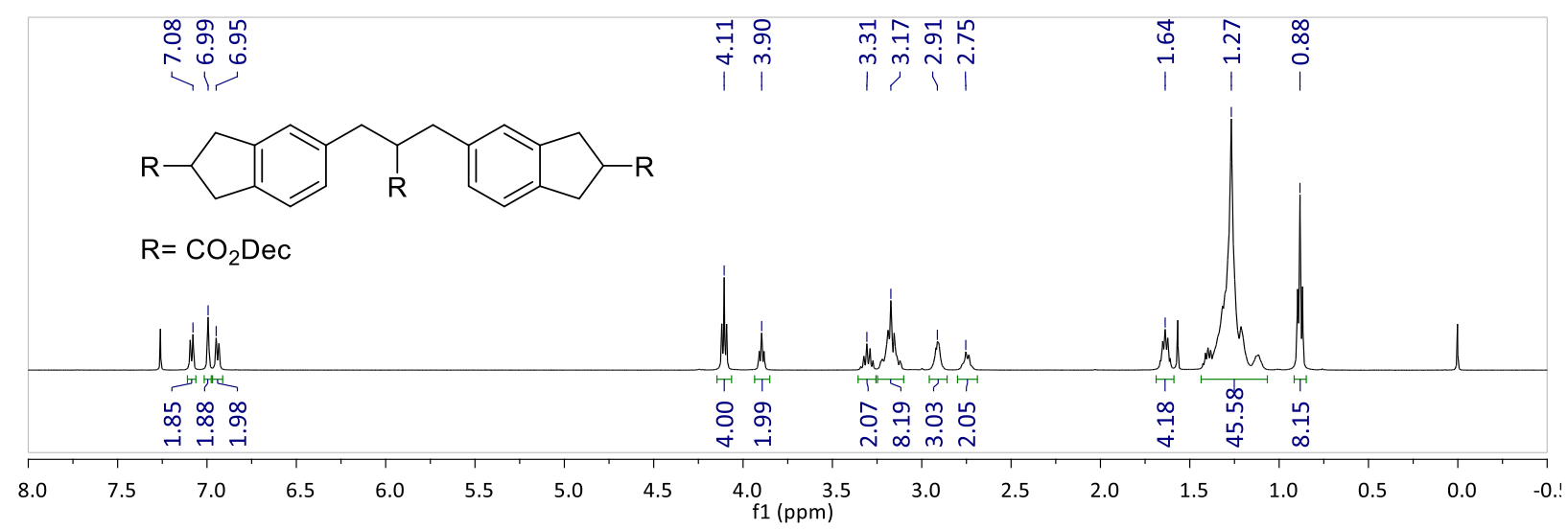


4f $\left({ }^{13} \mathrm{C} \mathrm{NMR}, \mathrm{CDCl}_{3}\right)$

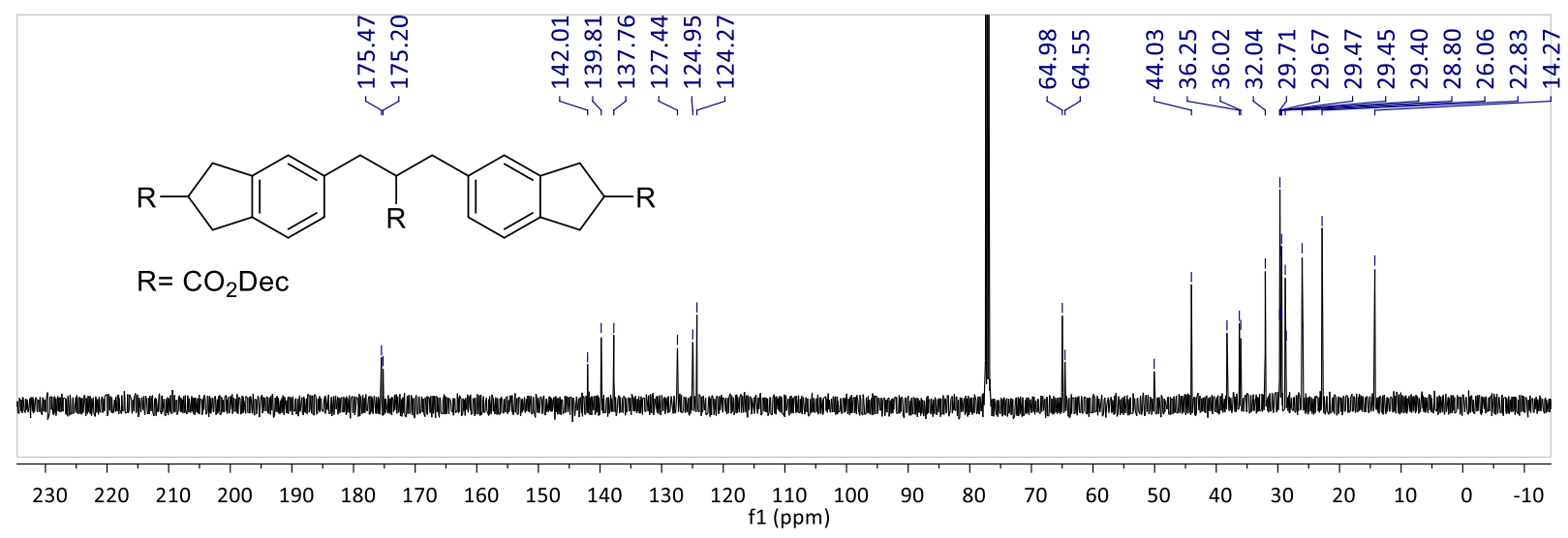




\section{Cross-over experiments}

\section{Reaction (I)}

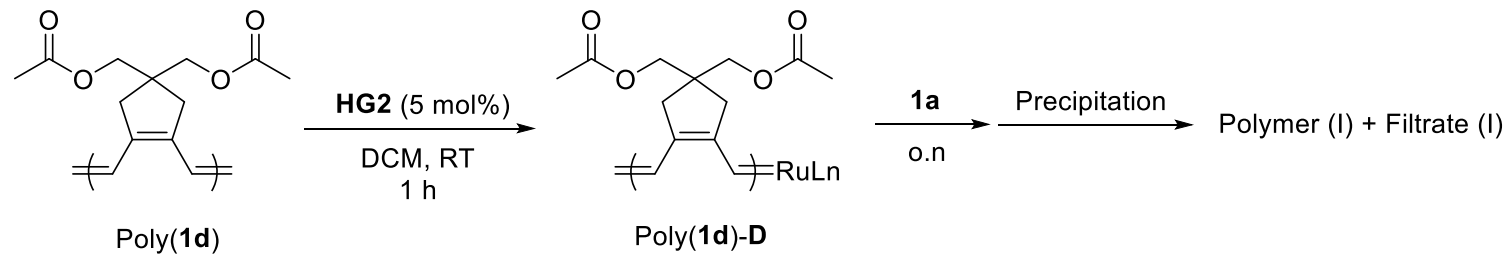

\section{Reaction (II)}
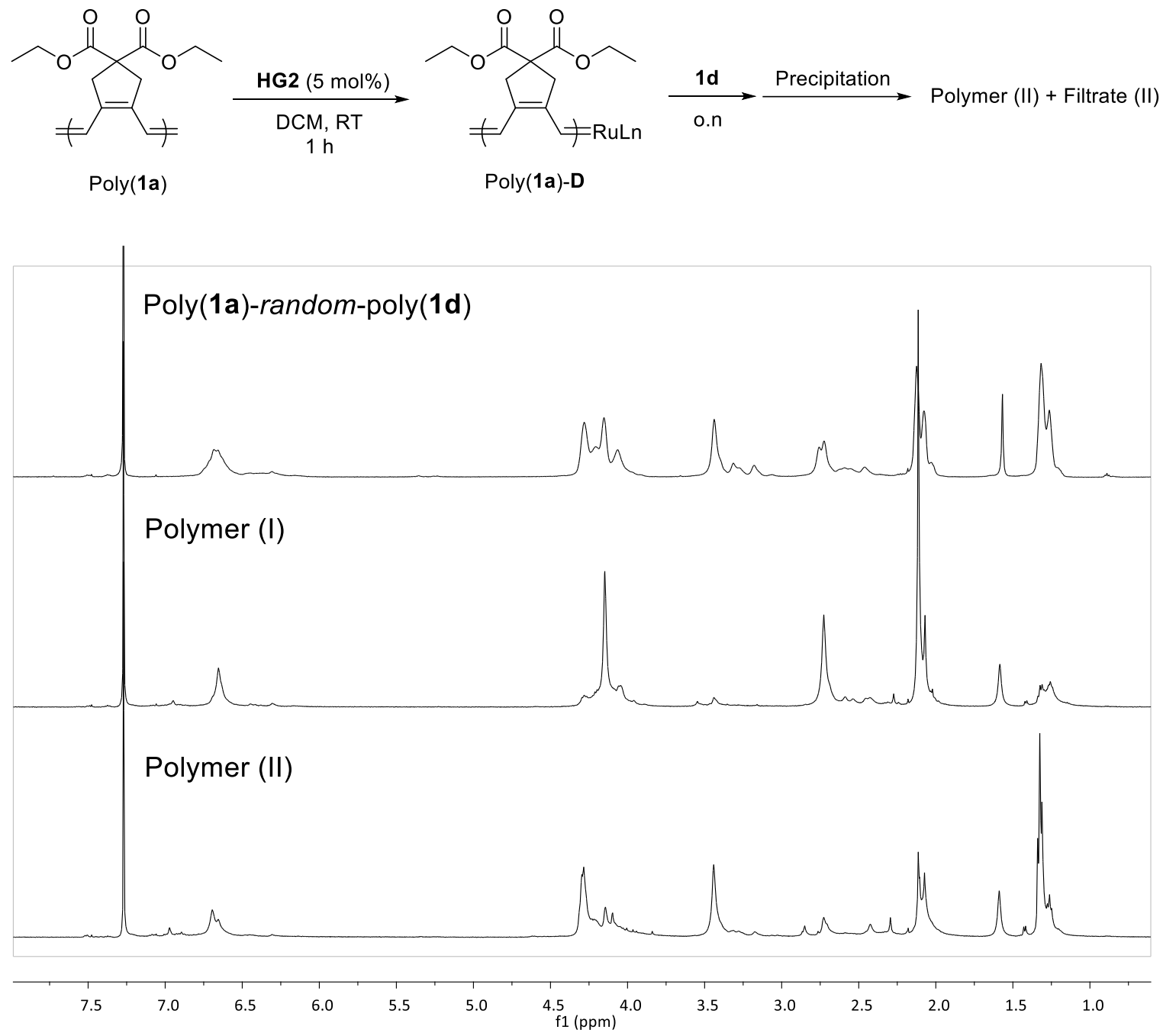

Figure S1. ${ }^{1} \mathrm{H}$ NMR of copolymer (poly(1a)-ran-poly(1d)), polymer (I), and polymer (II). 


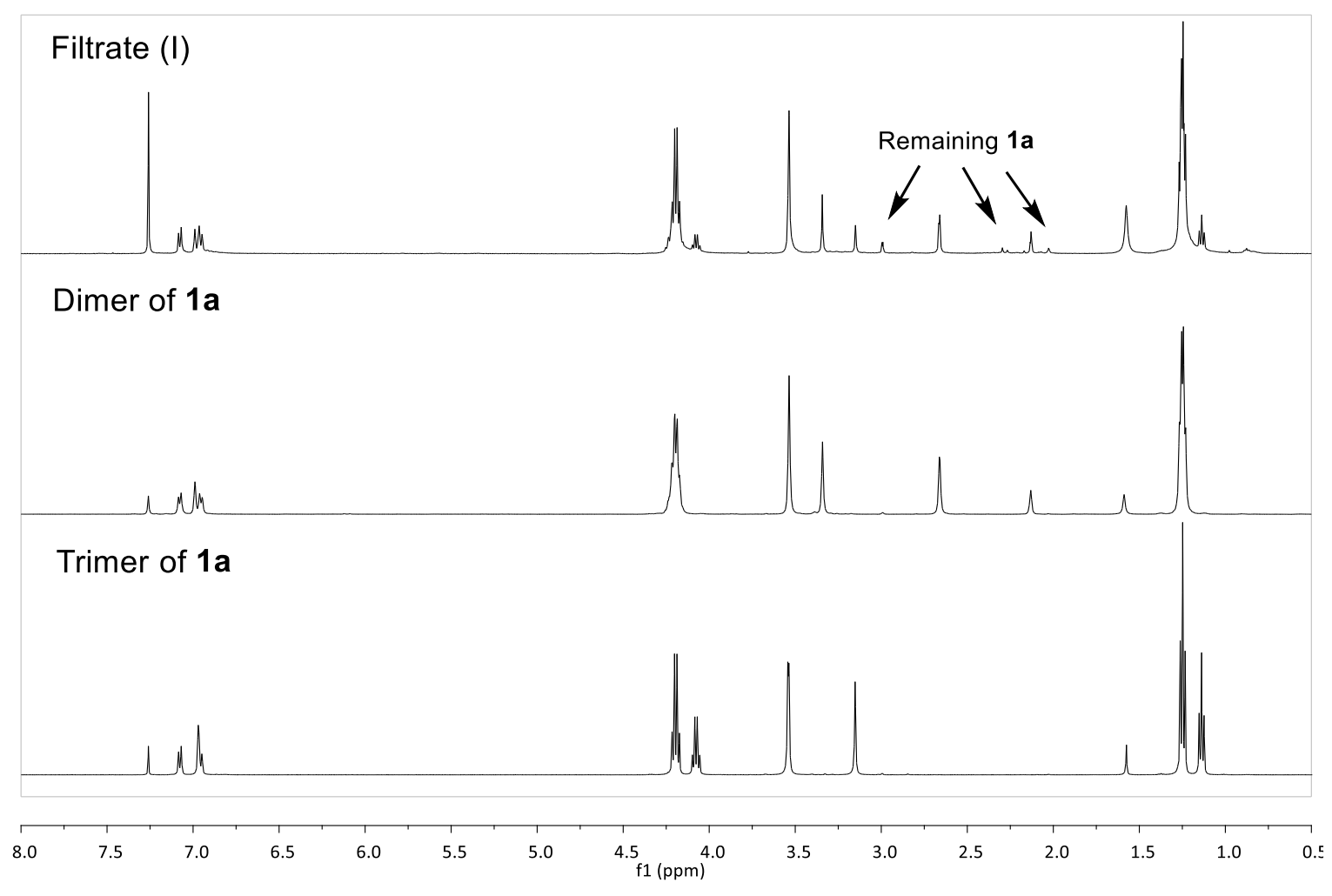

Figure S2. Filtrate from reaction (I) and dimer and trimer of 1a. 


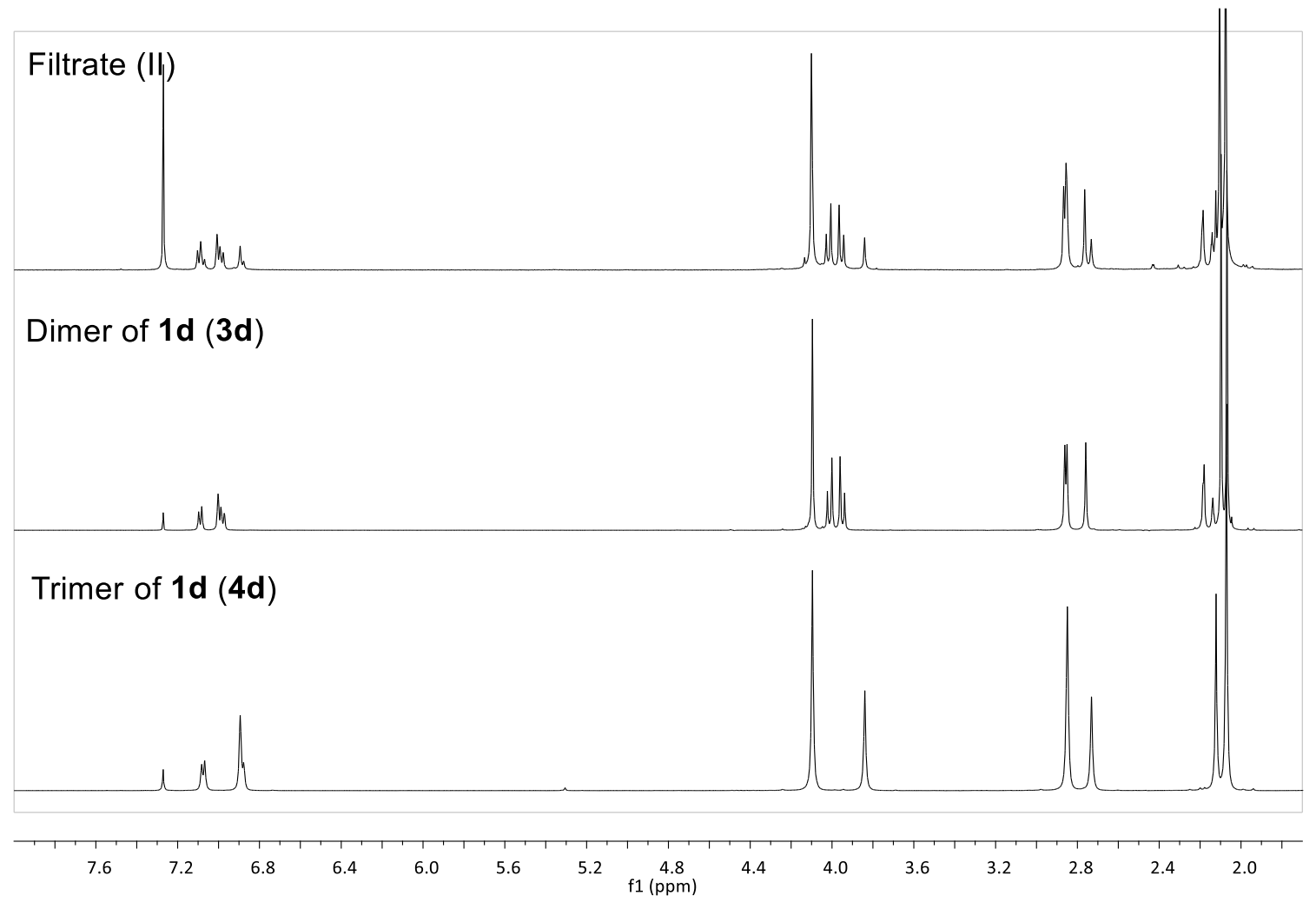

Figure S3. Filtrate from reaction (II) and dimer and trimer of $\mathbf{1 d}$. 
Characterization of mixture of 3a'-i and 3a'-ii

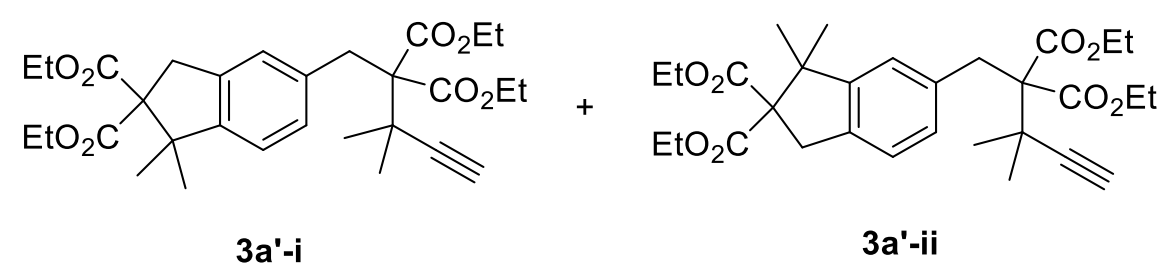

(1) ${ }^{1} \mathrm{H}$ and 2D NMR analysis
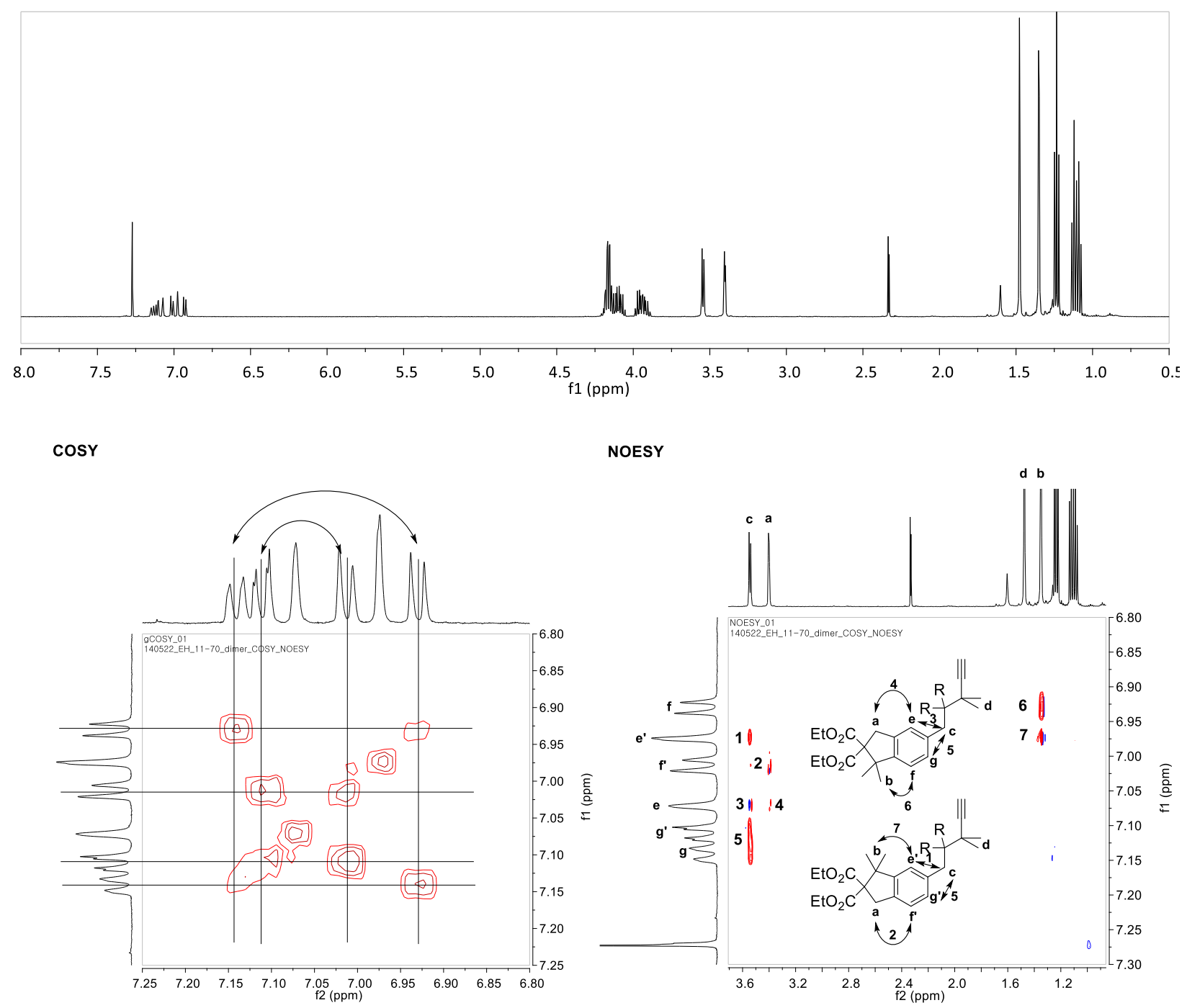

Figure S4. ${ }^{1} \mathrm{H}$ NMR and 2D NMR (COSY, NOESY) analysis 


\section{(2) GC/MS analysis}
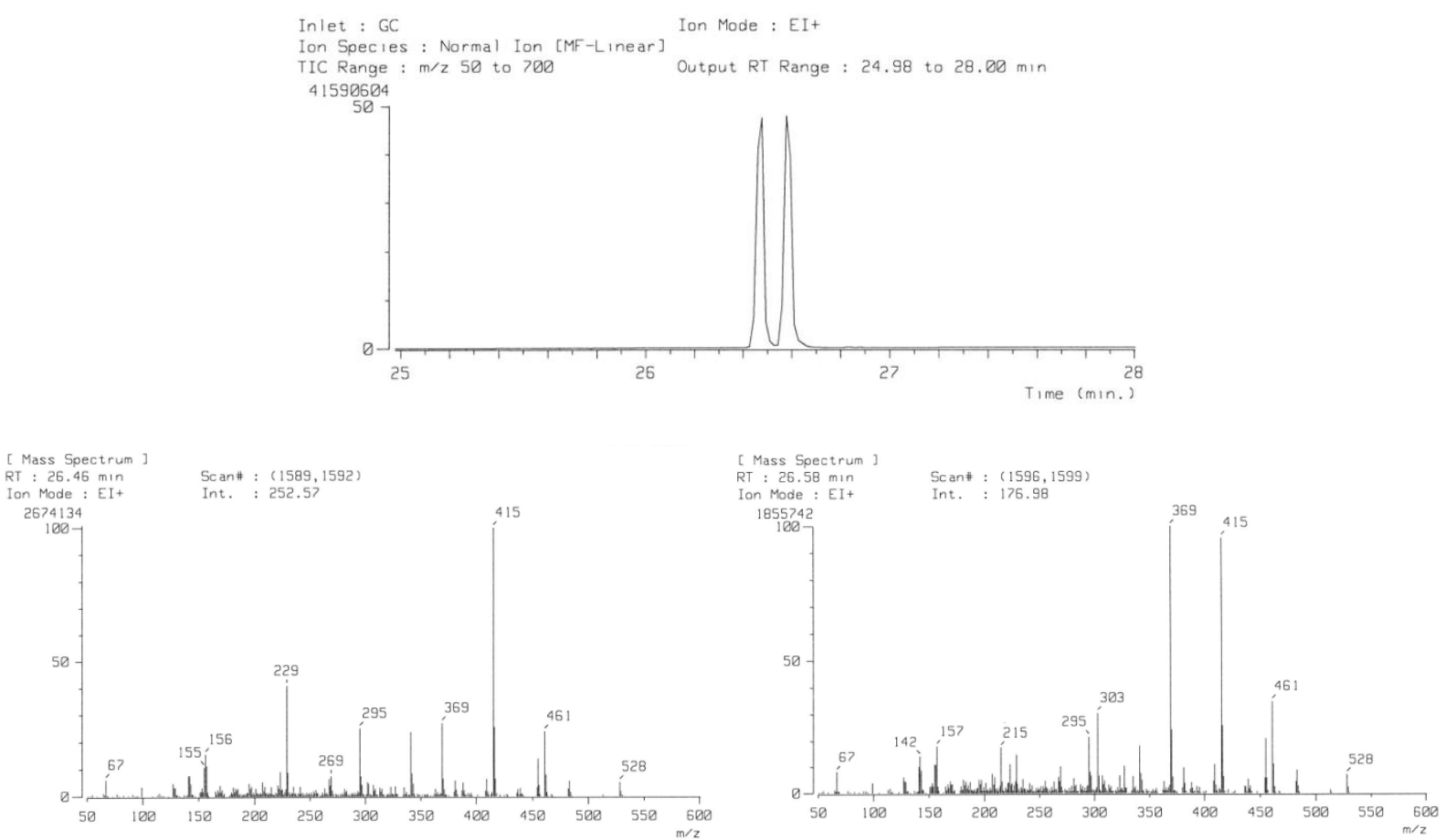

Figure S5. Gas chromatography of 3a'-i and 3a'-ii mixture and LRMS of each peak.

For 26.46 min; HRMS (EI+): calcd. for $\mathrm{C}_{30} \mathrm{H}_{40} \mathrm{O}_{8}, 528.2723$, found, 528.2717

For 26.58 min; HRMS (EI+): calcd. for $\mathrm{C}_{30} \mathrm{H}_{40} \mathrm{O}_{8}, 528.2723$, found, 528.2720

\section{Confirmation of the activity of ROMP by the decomposed catalyst}

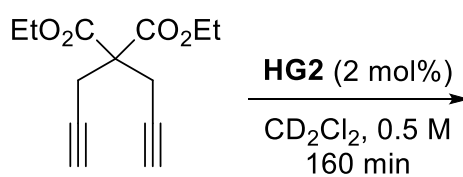

$[\mathrm{Ru}]$

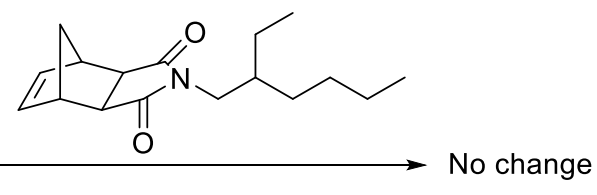

Figure S6. A norbornene derivative was added to the reaction mixture of 1a and HG2, and there was no ROMP of the norbornene after 30 minutes. It showed (i) the complete decomposition of the carbene and (ii) no reaction of the olefin by the decomposed catalyst. 


\section{Observation of the remaining carbene for the reactions of $1 \mathrm{e}-1 \mathrm{~h}$}

To a 2-mL vial were a monomer $(0.200 \mathrm{mmol})$ and magnetic bar added and purged with Ar. $400 \mu \mathrm{L}$ of $\mathrm{CD}_{2} \mathrm{Cl}_{2}$ was added to dissolve the monomer. After preparing a stock solution of initiator ( $35.8 \mathrm{mg}, 0.045 \mathrm{mmol}$ ) using $450 \mu \mathrm{L}$ of $\mathrm{CD}_{2} \mathrm{Cl}_{2}$ with a drop of hexamethyldisilane as an internal standard under the inert atmosphere, $100 \mu \mathrm{L}$ of the solution was ejected by microsyringe and transferred to Ar-filled NMR tube with septa cap, containing $450 \mu \mathrm{L}_{\text {of }} \mathrm{CD}_{2} \mathrm{Cl}_{2}$. ${ }^{1} \mathrm{H}$ NMR spectrum of it was obtained to check the initial ratio between the initiator and internal standard. To a solution of monomer, $100 \mu \mathrm{L}$ of the stock solution of initiator was added and stirred for $1-2$ minutes $(M / I=20)$, then the total volume of the reaction mixture $(500 \mu \mathrm{L})$ was rapidly transferred to Ar-filled empty NMR tube with a septa cap by syringe. ${ }^{1} \mathrm{H}$ NMR spectrum of the mixture was obtained with a scan number of 32 , and the remaining carbene signal was quantified by the internal standard. This process was repeated for $\mathbf{1 e}-\mathbf{1 h}$ using the same batch of the stock solution of the initiator.
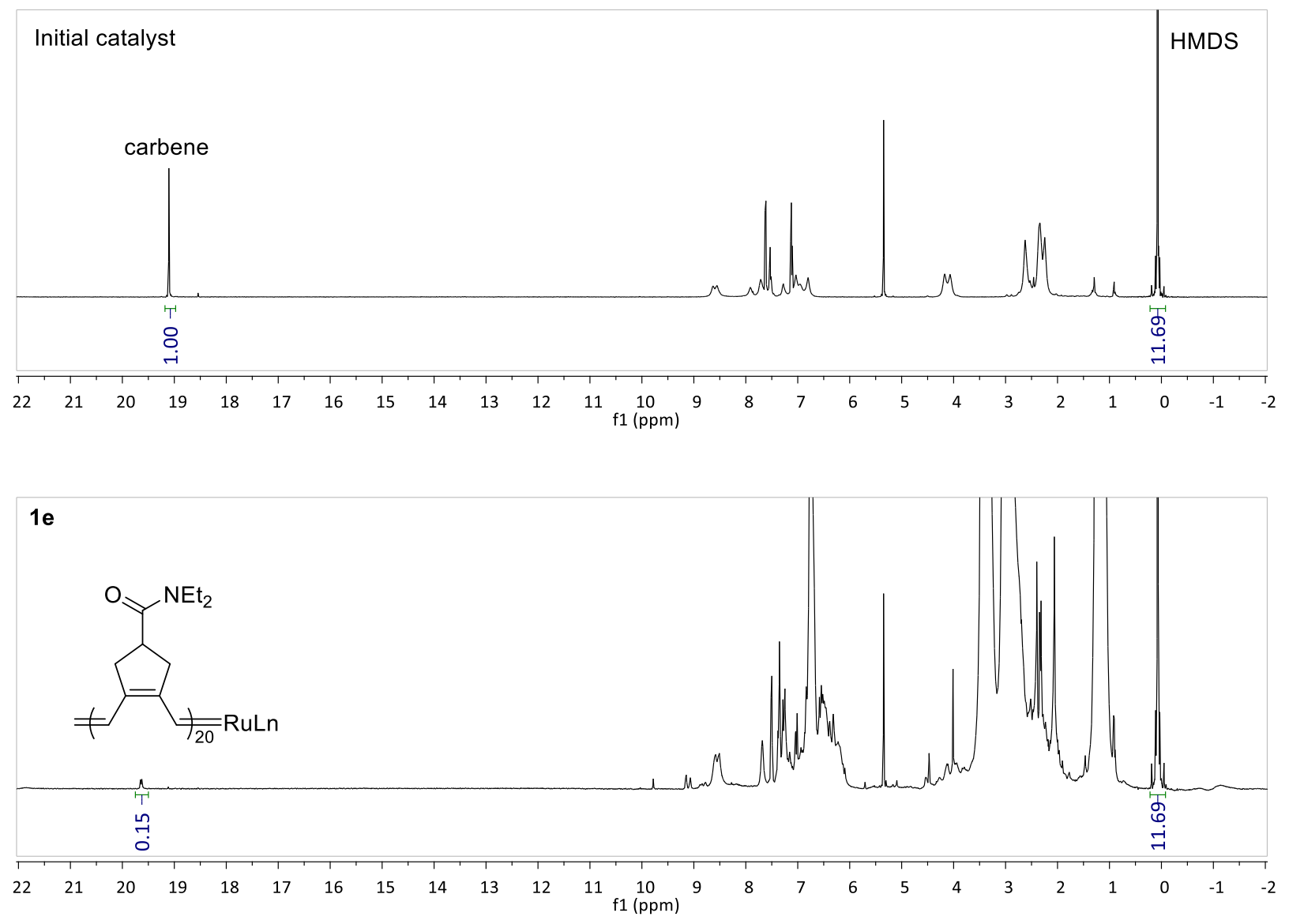

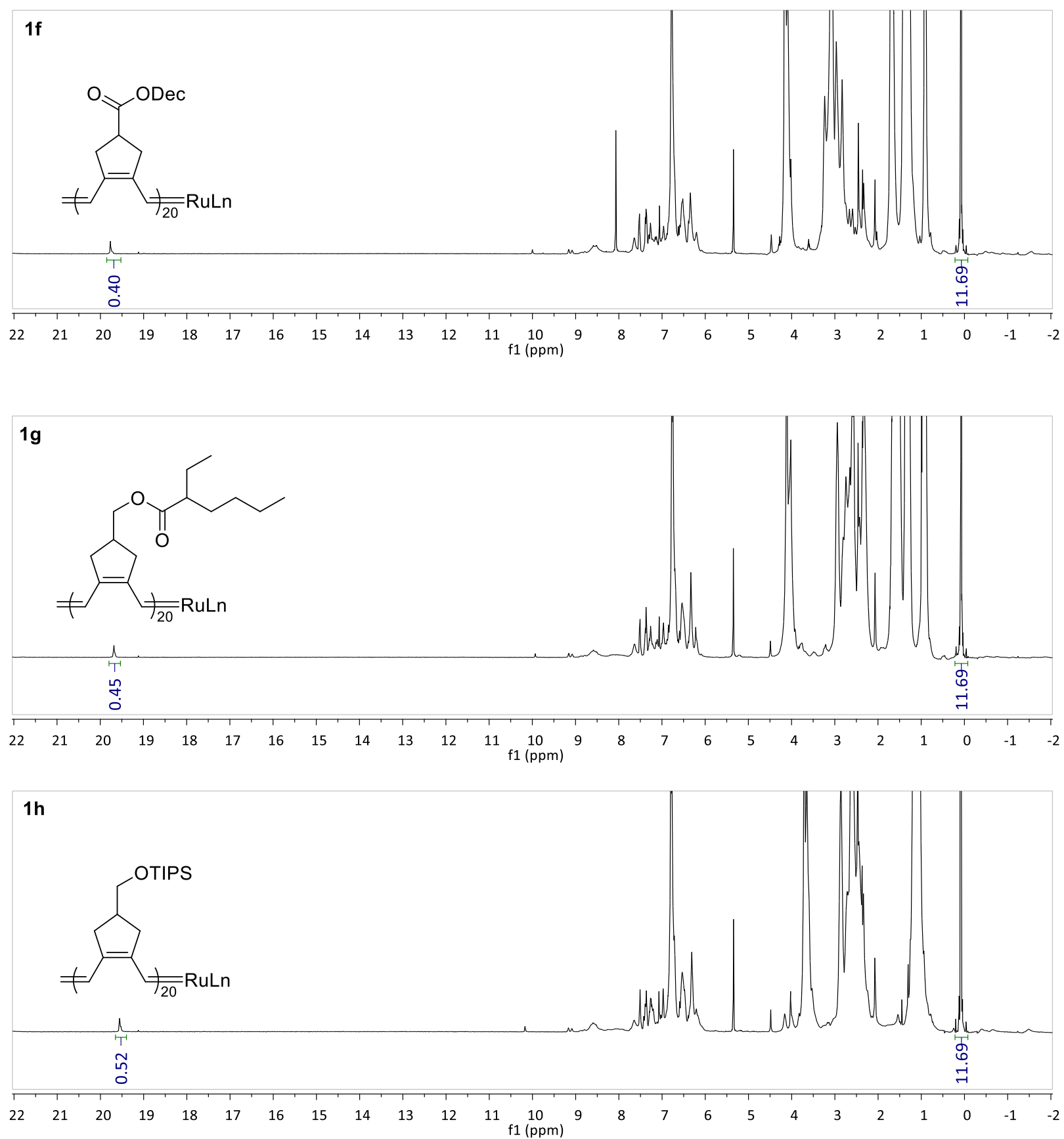

Figure S7. Quantitative analysis of remaining carbenes. 
Table S1. The addition of diethyl malonate to the CP of $\mathbf{1 h}$

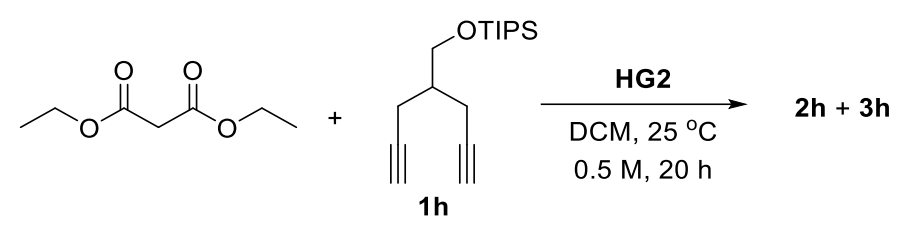

\begin{tabular}{|c|c|c|c|c|c|c|}
\hline entry & M:I:DEM & $\mathbf{2 h}$ & $\mathbf{3 h}$ & conv\% & $M_{\mathrm{n}}$ & PDI \\
\hline 1 & $25: 1: 10$ & Full & $\mathrm{X}$ & Full & $12.4 \mathrm{k}$ & 1.93 \\
\hline 2 & $25: 1: 25$ & Full & $\mathrm{X}$ & Full & $14.9 \mathrm{k}$ & 2.04 \\
\hline 3 & $25: 1: 100$ & Full & $\mathrm{X}$ & Full & $13.6 \mathrm{k}$ & 1.99 \\
\hline 4 & $25: 1: 250$ & Full & $\mathrm{X}$ & Full & $15.5 \mathrm{k}$ & 2.03 \\
\hline
\end{tabular}

\section{References}

(1) Anders, U.; Wagner, M.; Nuyken, O.; Buchmeiser, M. R. Macromolecules 2003, 36, 2668.

(2) Kang, E.-H.; Yu, S. Y.; Lee, I. S.; Park, S. E.; Choi, T.-L. J. Am. Chem. Soc. 2014, 136, 10508.

(3) Kang, E.-H.; Lee, I. S.; Choi, T.-L. J. Am. Chem. Soc. 2011, 133, 11904.

(4) Lee, I. S.; Kang, E.-H.; Park, H.; Choi, T.-L. Chem. Sci. 2012, 3, 761.

(5) Ungold, J.; Wang, D.; Frey, W.; Buchmeiser, M. R. Polym. Chem. 2013, 4, 4219.

(6) Saino, N,; Amemiya, F.; Tanabe, E.; Kase, K.; Okamoto, S. Org. Lett. 2006, 8, 1439.

(7) Sudheendran, M.; Horecha, M.; Kiriy, A.; Gevorgyan, S. A.; Krebs, F. C.; Buchmeiser, M. R. Polym. Chem. 2013, 4, 1590. 